\title{
Macro to nano specimen measurements using photons and electrons with digital holographic interferometry: a review
}

\author{
María del Socorro Hernández-Montes ${ }^{1 *}$, Fernando Mendoza-Santoyo ${ }^{1}$, Mauricio Flores Moreno ${ }^{1}$, \\ Manuel de la Torre-lbarra ${ }^{2}$, Luis Silva Acosta ${ }^{1}$ and Natalith Palacios-Ortega ${ }^{1}$
}

\begin{abstract}
Today digital holographic interferometry $(\mathrm{DH})$ is considered a modern full-field non-destructive technique that allows generating 3D quantitative data of a wide variety of specimens. There are diverse optical setups for DHI that enable the study of specimens in static and dynamic conditions: it is a viable alternative to characterize a wide diversity of parameters in the micro and macro world by conducting repeatable, reliable and accurate measurements that render specimen data, e.g., displacements, shape, spatial dimensions, physiological conditions, refractive indices, and vibration responses. This paper presents a review and progress on the most significant topics, contributions and applications involving DHI for the study of different specimens such as: cells, bio tissues, grains, insects, and nanostructures. For most of the research work involving macro and micro specimens the wave-like source used in the measurements were photons from a laser, while the studies carried out in the nano regime used the wave-like nature of the electron.
\end{abstract}

Keywords: Digital holographic interferometry; macro to nano measurements; bio-applications, Cells, Tissues, Electrons, Photons

\section{Introduction}

Dennis Gabor reported the invention of Holography in 1949. His main concern being the aberration correction in the electron microscopes. At the time the lack of coherent electron sources meant that the hologram reconstruction was done using quasicoherent light sources. As such Holography did not produce enough results to be considered a must use tool, even though a device called a wire-biprism was invented to combine the object and reference beams.

\footnotetext{
*Correspondence: mhernandez@cio.mx

${ }^{1}$ Centro de Investigaciones en Óptica A. C, (CIO), Loma del Bosque 115, León, 37150 Guanajuato, Mexico

Full list of author information is available at the end of the article
}

The invention of the laser at the end of the 1950's gave a great leap to Holography since this light source was highly coherent and hence led to the invention of Holographic Interferometry (HI) during the first lustrum of the 1960's. A seminal manuscript that introduces the concept of $\mathrm{HI}$ was written by Powell and Stetson, who described the technique as one furthering the studies of the Leith-Upatnieks hologram for the time average of coherent wavefronts scattered by a vibrating object [1]. The hologram was recorded on a photographic plate that had to undergo the chemical process needed to develop and fix the film. A step forward in the development of $\mathrm{HI}$ was the electronic detection of holograms reported by Goodman and Lawrence [2], for computer synthesis holograms [3], and in the earlier work reported by Brown, et al. [4]. Perhaps the first manuscript that coined the term holographic interferometry was published in 
1966 by Heflinger et al. [5], who reported the wellknown fact that the holographic technique of photography is founded in the interference phenomena and hence the double exposure technique to obtain interference fringes in the holograms. Digital Holography (DH) takes over conventional Holography when digital recording of the holograms is achieved with electronic devices such as CCD sensors instead of the so called holographic film emulsion. One of the main advantages of the $\mathrm{DH}$ recording is that data can be stored allowing for the numerical reconstruction of both the intensity and phase of the object wavefront. As reported by Schnars et al. [6, 7], digital recording and numerical reconstruction of holograms started with the first publications on the field including not only digital recording of holograms by an electronic camera, but also the implementation of basic diffraction algorithms to numerically reconstruct the intensity and phase information embedded in a hologram. After these first communications, many researches linked the term Digital Holographic Interferometry (DHI) to the process of digitally recording a hologram, and the use of numerical algorithms based on classical diffraction theory to get the intensity and phase information imbedded in the holograms [8]. DHI directly derives from DH as conventional holographic interferometry naturally derived from holography during the middle of the 1960's decade; DHI allows the measurement of the object amplitude and phase from objects that undergo static or dynamic changes in time. Thus, it is used to record two different states of the object, and permits various measurements from the phase variations, for example, surface deformation, surface shape, refractive index, vibration conditions, etc. Furthermore, DHI also refers to the numerical processing of the holograms to get intensity and phase information by spatial filtering in the Fourier domain [9-11].

In DHI an interference image formed by the overlapping of the diffracted object beam and the reference beam is captured and recorded at the location of the digital sensor (today usually a CCD, or the like, camera). The amplitude and phase of the object are encoded into the intensity of the hologram. Currently, DHI used with photons is widely applied in a quantitative and qualitative manner to perform macro or micro measurements, for instance, in the physical, mechanical and physiological characterization of parameters from ample and diverse specimen types. This discipline in the Optics field has successfully evolved to become a trusted tool in a wide variety of areas.

Electrons also behave as waves and thus can create interferograms. Reliable and highly coherent electron sources were made available by the late 1970's, a fact that also gave an outstanding impulse to electron holography not only due to the coherent field electron guns (feg) used in the hologram reconstruction process but also because of the appearance of electron holographic interferometry (EHI) as a "quasinon-invasive" measurement tool in transmission electron microscopes (TEM), set in the so called holography mode. Today nanomaterials and structures belonging to a wide variety of areas can be characterized in regards to their physical and mechanical parameters using $\mathrm{HI}$ with light sources and EHI. Indeed, many reconstruction processes learnt with DHI using photons can be directly applied to EHI. For most of the specimen characterization involving micro to nano work, the source used in the measurement systems were either photons from a laser or electrons from a feg.

In this paper, we will review and discuss the state of the art in DHI and EHI applications to study the displacements, shape, spatial dimensions, physiological conditions, refractive indices and vibration responses of different samples as bio tissues, grains, insects, nanoparticles, cells, and bacteria, among others. These examples show that both DHI and EHI have become a reliable and robust metrology tool, gradually used in the domain of biological/medical applications.

\section{Digital holographic interferometry (DHI)}

Digital Holographic interferometry (DHI) provides an interferometric comparison of objects or events separated in time and space. In DHI, the holographic images are formed from the superposition of the reference and object waves: one image corresponds to the object in a known base state while a second image is acquired when the object has undergone a displacement or deformation. Both images are recorded on the CCD sensor and stored in the PC hardware for further manipulation with the aim of recovering the amplitude and phase that result from the object change in its base and deformed states. DHI is a full-field-of-view technique that has been applied to investigate a wide variety of objects and numerous unique applications. Depending on the application DHI uses cw or pulsed lasers, and can be configured in either mode: transmission, for instance to look at transparent objects (such as cells) and obtain refractive index changes or sample thickness [12]; or scattering (diffuse or nonspecular objects), to obtain displacement, deformation or rotation information from the object [13].

\section{Optical schematics of DHI}

A typical arrangement for transmission mode to study phase objects is shown in Fig. 1a. The optical layout 


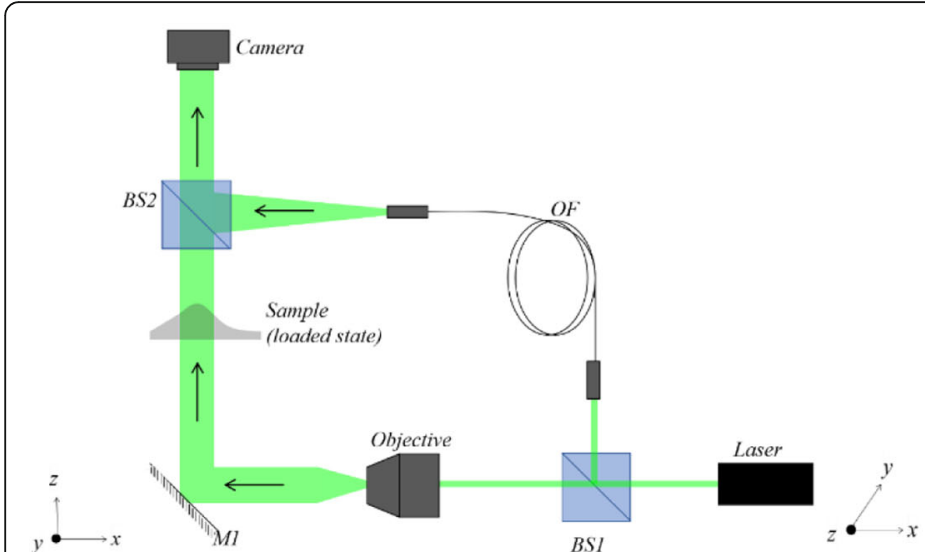

(a)

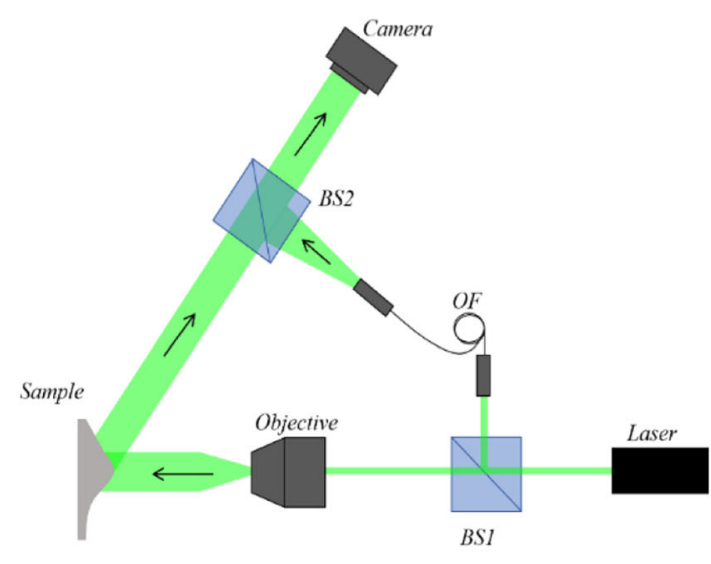

(b)

Fig. 1 a Holography Interferometry for phase objects, and $\mathbf{b}$ Holography Interferometry for opaque objects

is that of a Mach Zehnder interferometer, where a laser is divided into reference and object waves by means of a beam splitter (BS1). The reference wave is guided through an optical fiber (OF), while the object wave is expanded with a microscope objective and a mirror (M1) directs the beam through the sample and with the help of another beam splitter, (BS2), the reference and object waves are combined to form an interference pattern (also called an image hologram) on a CCD sensor. The arrangement for diffuse objects is depicted in Fig. 1b, its image acquisition procedure is the same as for phase objects. The optical fiber in both arrangements can be replaced with a mirror to guide the reference beam, however, recently the use of the optical fibers has allowed simpler arrangements for holography interferometry, and also avoid the use of lenses to manipulate the size of the reference beam. Nowadays, systems that integrate digital camera's sensors, optical fibers, and even piezoelectric fiber-optic modulators are known as Optoelectronic Holography Systems [14]. Usually for both arrangements an aperture is located right before the second beam splitter (BS2). Its use helps to increase the imaging system depth of focus, controls the amount of light that reaches the sensor, and serves as an object image band pass filter. The latter is further used during the holographic image processing that involves data manipulation in the Fourier space, where image data is spatially filtered in order to finally obtain object's amplitude and phase. Numerically the extraction of the phase information is usually made according to the Fourier-Takeda algorithm [9-11]. This procedure is detailed in the next section.

\section{Working principle}

Briefly, in mathematical terms the DHI method to retrieve the optical phase is based on the interaction of a smooth reference wave and an object wave expressed as $R$ and $O$ respectively. The intensity recorded on the digital camera's sensor for each image hologram is given by;

$$
\begin{aligned}
I\left(x_{H}, y_{H}\right) & =\left|R\left(x_{H}, y_{H}\right)\right|^{2}+\left|O\left(x_{H}, y_{H}\right)\right|^{2} \\
& +R\left(x_{H}, y_{H}\right) O^{*}\left(x_{H}, y_{H}\right) \\
& +R^{*}\left(x_{H}, y_{H}\right) O\left(x_{H}, y_{H}\right)
\end{aligned}
$$

where $x_{H}$ and $y_{H}$ are the $x$ and $y$ coordinates at the image hologram plane $H$ (sensor) and the * denotes the complex-conjugate amplitude. The last two terms of Eq. (1) contain information that corresponds to the amplitude and the phase of the object wave, data which is retrieved using the Fast Fourier transform method [9-11]. Expressing the object and the reference waves in terms of amplitude and phase results in:

$$
\begin{aligned}
& O\left(x_{H}, y_{H}\right)=o\left(x_{H}, y_{H}\right) e^{i \phi\left(x_{H}, y_{H}\right)} \\
& R\left(x_{H}, y_{H}\right)=r\left(x_{H}, y_{H}\right) e^{-2 \pi i\left(f_{x} x_{H}+f_{y} y_{H}\right)} .
\end{aligned}
$$

Where $o$ and $r$ are the object and reference amplitudes respectively, and $f_{x} x_{H}$ and $f_{y} y_{H}$ represent the spatial carrier frequencies along $x_{H}$ and $y_{H}$ coordinates. Substituting these terms in eq. 1 gives, 


$$
\begin{aligned}
I\left(x_{H}, y_{H}\right)= & a\left(x_{H}, y_{H}\right)+c\left(x_{H}, y_{H}\right) e^{2 \pi i\left(f_{x} x_{H}+f_{y} y_{H}\right)} \\
& +c^{*}\left(x_{H}, y_{H}\right) e^{-2 \pi i\left(f_{x} x_{H}+f_{y} y_{H}\right)}
\end{aligned}
$$

With,

$$
\begin{aligned}
& a\left(x_{H}, y_{H}\right)=o^{2}\left(x_{H}, y_{H}\right)+r^{2}\left(x_{H}, y_{H}\right) \\
& c\left(x_{H}, y_{H}\right)=o\left(x_{H}, y_{H}\right) r\left(x_{H}, y_{H}\right) e^{i \phi\left(x_{H}, y_{H}\right)}
\end{aligned}
$$

The last two terms of equation (2) indicate where the spatial carrier is phase modulated by the term $\phi\left(x_{H}, y_{H}\right)$ introduced due to the interference signal. These spatial carrier is introduced in the reference beam due to a small inclination with respect to the observation axis of the optical system. In order to retrieve the optical phase, equation 2 is Fourier transformed in two dimensions,

$$
\begin{aligned}
F T\left\{I\left(x_{H}, y_{H}\right)\right\}= & A\left(f_{x_{H}}, f_{y H}\right) \\
& +C\left(f_{x_{H}}-f_{x} x_{H}, f_{y H}-f_{y} y_{H}\right) \\
& +C^{*}\left(f_{x_{H}}+f_{x} x_{H}, f_{y H}+f_{y} y_{H}\right) .
\end{aligned}
$$

The $C$ and $C^{*}$ terms are the Fourier transform of $c$ and $c^{*}$ respectively (each contains the same phase information $\left.\phi\left(x_{H}, y_{H}\right)\right)$ while $A\left(f_{x_{H}}, f_{y H}\right)$ is the lowfrequency background illumination, and $\left(f_{x_{H}}, f_{y H}\right)$ is the frequency coordinate. As they are spatially separated in the Fourier spectrum a bandpass filter is used to eliminate the $A$ and $C^{*}$ terms. The $C$ term is frequency centered and an inverse Fourier transform is applied to each filtered image hologram. Then, the phase can be obtained with

$$
\phi\left(x_{H}, y_{H}\right)=\arctan \frac{\operatorname{Imc}\left(x_{H}, y_{H}\right)}{\operatorname{Rec}\left(x_{H}, y_{H}\right)},
$$

where $\operatorname{Im} c\left(x_{H}, y_{H}\right)$ and $\operatorname{Re} c\left(x_{H}, y_{H}\right)$ are the imaginary and real parts of the Fourier inverted hologram with the $C$ term. Upon subtracting the two phases for the base $(\phi)$ and deformed $\left(\phi^{\prime}\right)$ object states, a wrapped phase map is obtained showing the relative phase difference $(\Delta \phi)$ calculated with

$$
\Delta \phi\left(x_{H}, y_{H}\right)=\phi\left(x_{H}, y_{H}\right)-\phi^{\prime}\left(x_{H}, y_{H}\right) \text {. }
$$

Equation 6 could be expressed in terms of $c$ and $c^{\prime}$ before and after the object's deformation as follows,

$$
\Delta \phi\left(x_{H}, y_{H}\right)=\frac{\operatorname{Re}\{c\} \operatorname{Im}\left\{c^{\prime}\right\}-\operatorname{Re}\left\{c^{\prime}\right\} \operatorname{Im}\{c\}}{\operatorname{Re}\{c\} \operatorname{Re}\left\{c^{\prime}\right\}+\operatorname{Im}\{c\} \operatorname{Im}\left\{c^{\prime}\right\}}
$$

The relative optical phase difference can be related to a vector displacement $(\vec{U}), \Delta \phi=\vec{k} \cdot \vec{U}, \vec{k}=\widehat{r_{H}}-\widehat{r_{s}}$ [13], where $\vec{k}$ is the sensitivity vector which changes according to the experimental set up, $\widehat{r_{H}}$ and $\widehat{r_{S}}$ are the unit vectors of observation and illumination, respectively. For an outof-plane configuration the interferometer's sensitivity is along the $z$ axis (i.e., the observation direction), hence

$$
u_{z}=\frac{\Delta \phi \lambda}{2 \pi(\cos \beta+1)}
$$

$u_{z}$ represents the variable for the out-of-plane displacements in the $z$ direction, $\beta$ is the angle between the

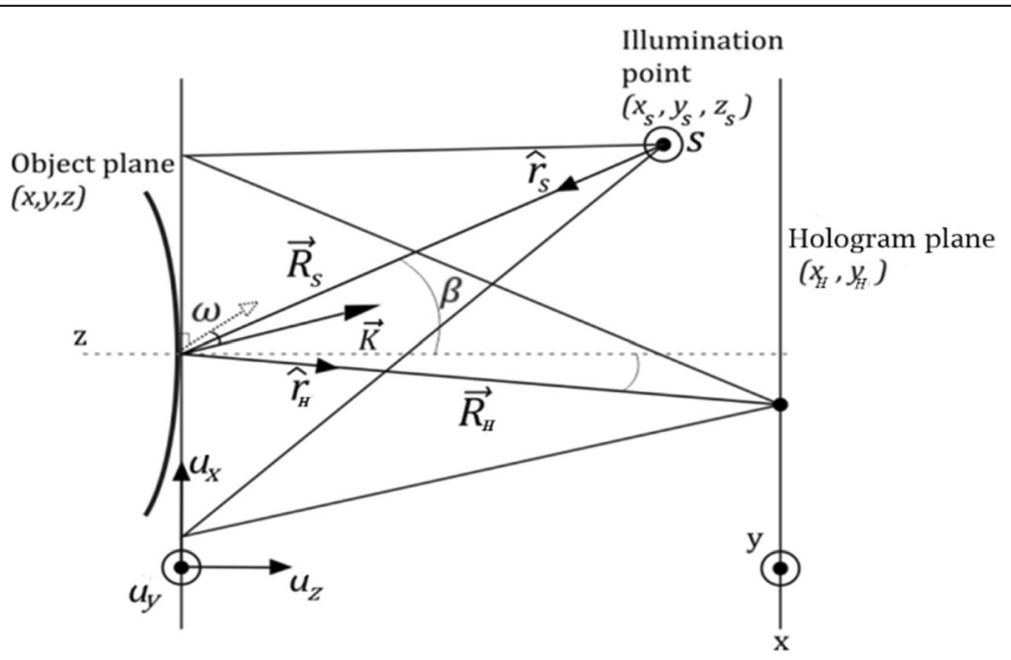

Fig. 2 The layout of Out-of-plane displacements setup. An object plane at $(\boldsymbol{x}, \boldsymbol{y}, \boldsymbol{z})$ is illuminated by $S$ light source at illumination point $\left(\boldsymbol{x}_{\boldsymbol{s}}, \boldsymbol{y}_{\boldsymbol{s}}, \boldsymbol{z}_{\boldsymbol{s}}\right)$, with direction represented by $\overrightarrow{\boldsymbol{R}_{\boldsymbol{S}}}$ vector. The light scatters reaches to the hologram plane (observation plane) at $\left(\boldsymbol{x}_{\boldsymbol{H}}, \boldsymbol{y}_{\boldsymbol{H}}\right)$, with direction represented by $\overrightarrow{\boldsymbol{R}_{\boldsymbol{H}}}$ vector 
object illumination and the observation directions. Please notice that equation 8 is a reduced expression valid for smooth surfaces and small illumination angles.

For more accurate out-of-plane displacements measurements, the angle $\omega$ given by the geometry of the object is introduced in the previous equation, see Fig. 2, i.e., it is the angle between the sensitivity vector and the normal to the surface [15], then

$$
u_{z}=\frac{\Delta \phi \lambda}{2 \pi(\cos \beta+1) \cos \omega}
$$

\section{Biological application using DHI}

The application of DHI in biomedical objects has increased significantly during the last two decades, mainly due to the development of new semiconductor devices that have had an impact on new and improved electronic devices such as illumination sources, imaging devices and so on. Normally, a biological sample requires extensive hardware dedicated to contain, preserve and manipulate it during the optical inspection. For these reasons it is notorious the complexity in the reported experimental setups when biological samples are studied with interferometric techniques. One knew and extensive tool for these inspections is the use of stroboscopic illumination at fast repetition rates [16], a tool that helps to reduce the error (de-correlated optical phase) of the measurements due to the sample's motion. This strobed illumination is possible with the use of a pulsed laser $[17,18]$ synchronized with the camera's acquisition software. This characterization includes qualitative imaging and quantitative measurements of form, geometry, displacement, vibration, stress, strain and viscoelastic properties of several objects going from handmade manufactured materials to biological and tissue samples.

The analysis of shape and several other parameters in transparent media $[19,20]$ is one of the first approximations to the biological inspection as the size of some samples is nearly invisible for a regular size inspection. The transparent inspection principle is tested in liquid media where the optical technique is able to retrieve a smooth and useful optical phase, which is used to measure different parameters such as temperature [21] and liquid interactions [22, 23]. In reference [24] a study using a gas-liquid interface is presented, where the authors observe the changes in the liquid phase due to the mass transfer process coupled with chemical reactions. Figure 3 shows the experimental cell used during this test while Fig. 4 shows the fringe patterns before and after the transfer. Observing Fig. 4 it is possible to detect the chemical reaction due to the $\mathrm{CO}_{2}$ absorption which induces refractive index variations in the liquid below the interface.

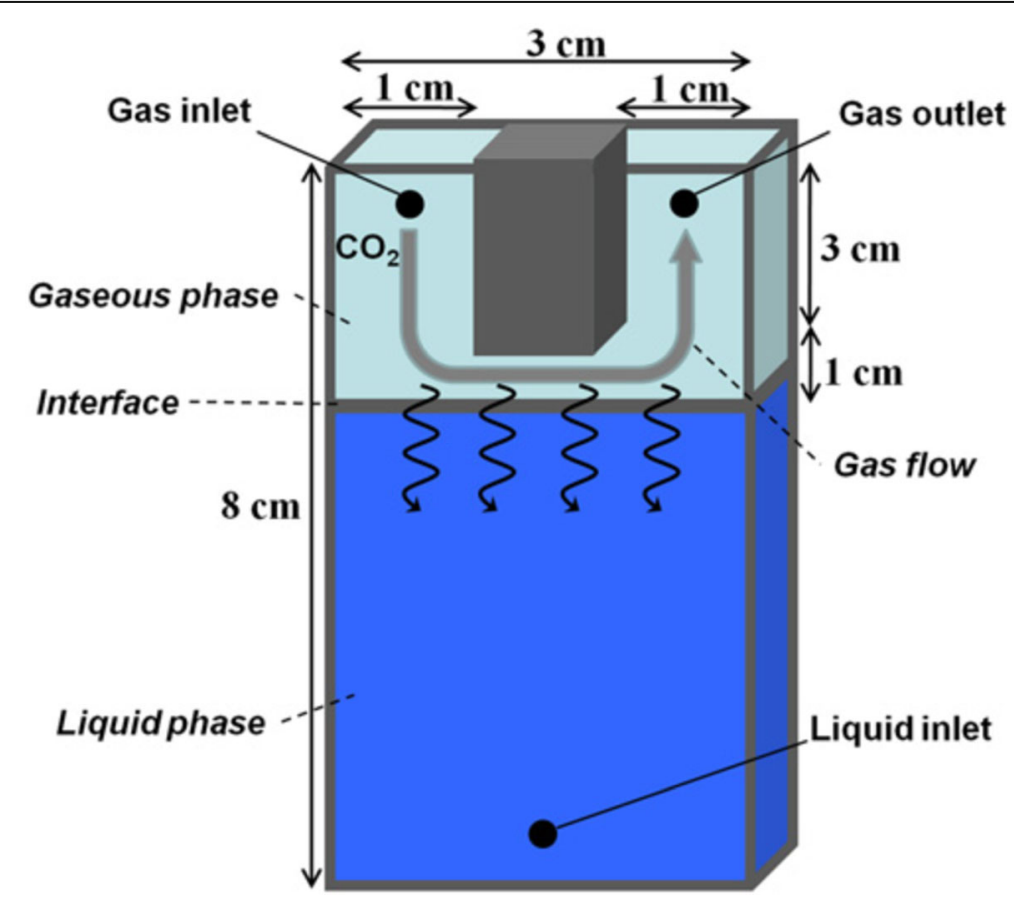

Fig. 3 Block-diagram of the experimental cell. Reprinted from [24]. Copyright [2011], ELSEVIER 


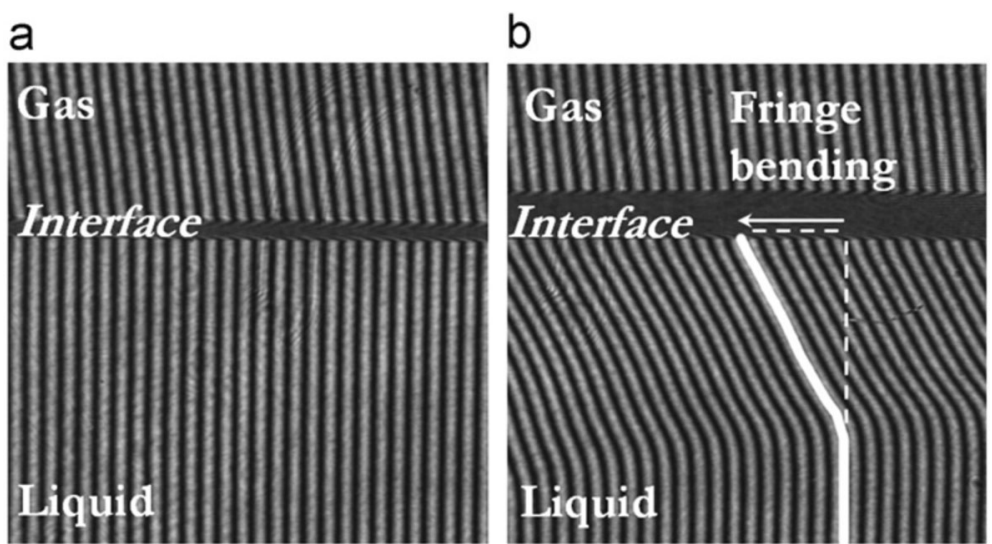

Fig. 4 Interferograms (a) before the beginning of the transfer and (b) 15 min after. Reprinted from [24]. Copyright [2011], ELSEVIER

The inspection in different media helps to better understand complex interactions that are common in biological tissues when they change under no control. This complex behavior is explored in reference [25] using a high-speed DHI configuration. The samples under study are bird feathers in-vivo (the living bird is actually inspected, without suffering harm). Each feather is an intricate collection of hundreds of barbules and hooks interconnected and supported by barbs to the rachis (see Fig. 5). This makes a flexible ensemble that is always in motion and continuously modifying its direction by the bird's movement, its blood flow, and the barbules' orientation. The latter is a challenging inspection if a large area of the bird needs to be illuminated in order to retrieve a smooth optical phase map (as those shown in Fig. 6). The use of high-speed hardware helps to avoid the slow motion of the bird, however the feather orientation is a different matter and specific algorithms were designed to match the phase maps with the bird's surface texture without de-correlation, see Fig. 7. In this kind of application, it is notorious the adaptability of DHI dealing with non-repeatable deformations in harsh conditions.

There are much more biological inspections in living organisms using DHI, but we recommend particular attention to the ones reported for the case of insects (butterflies) for wing flapping studies [26] and wing flapping comparison [27].

Another interesting example is described in reference [28] where the skin is studied, a biomedical subject that demands alternative inspection tools. The skin elasticity is one of the most studied mechanical properties because it plays an important role in healthy and unhealthy skin [28-31]. There are several physiological processes and diseases that can be directly correlated with changes in elasticity [32, 33], e.g., skin cancer presents severe changes in elasticity meaning that it is a useful parameter to study in order to better understand the mechanisms of this illness [34]. The work in reference [28] presents the variations in porcine skin rigidity caused by exposure to UV radiation, studied using a digital holographic interferometer in scattering mode. Displacement fields

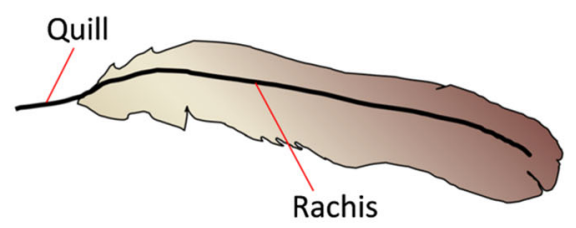

(a)

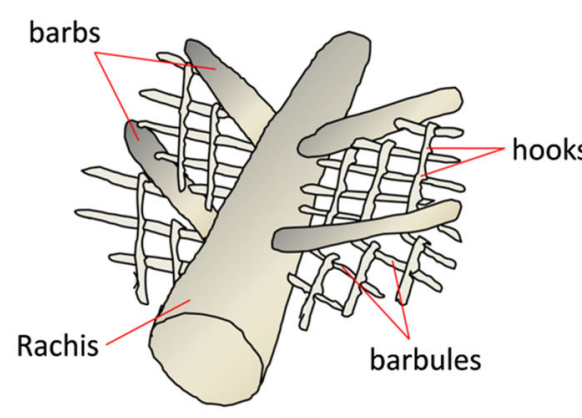

(b)

Fig. 5 Schematic view for: (a) primary feather and (b) feather central section. Reprinted from [25]. Copyright [2013], SPIE 

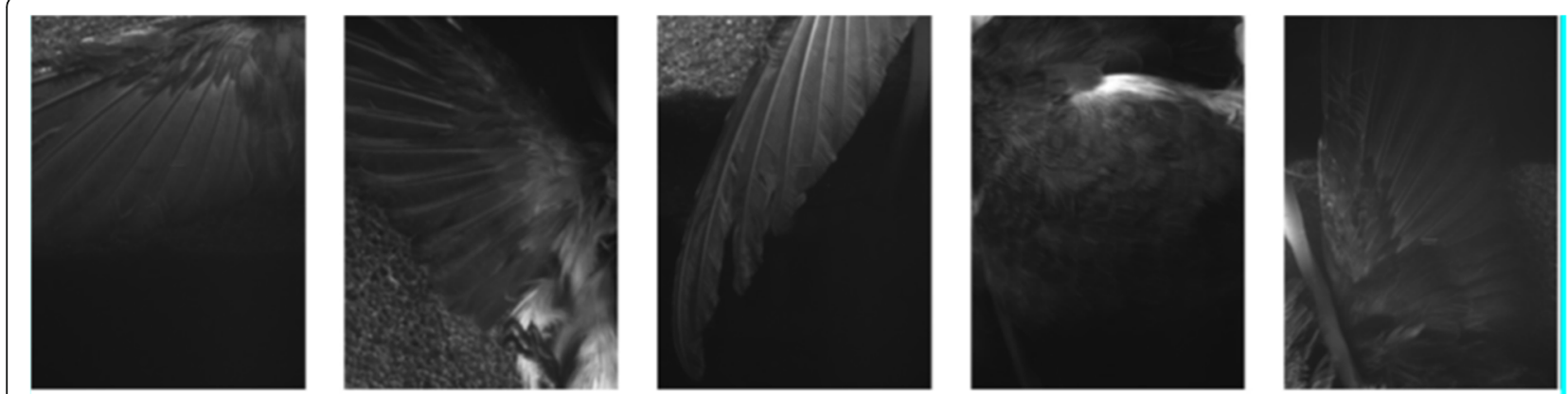

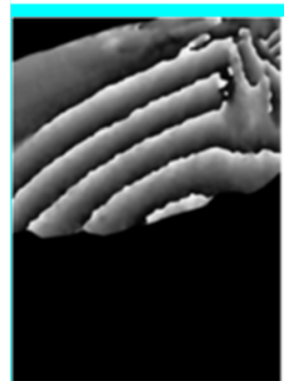

(a)

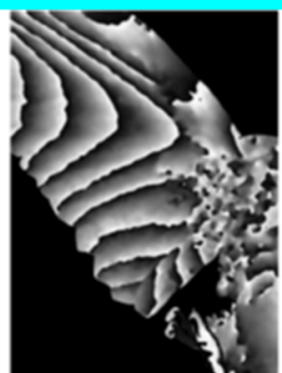

(b)

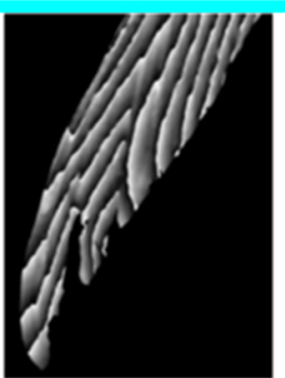

(c)

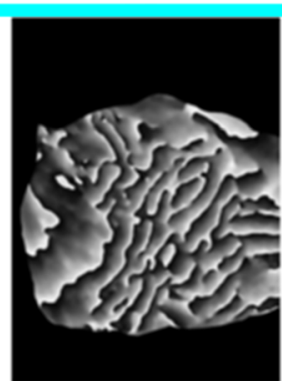

(d)

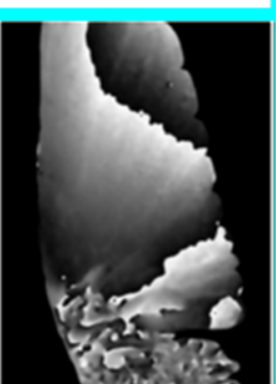

(e)

Fig. 6 Examples of retrieved phase maps from different bird's body sections. Reprinted from [25]. Copyright [2013], SPIE

normal to the surface of the skin under observation were obtained using DHI. To induce $\mathrm{z}$-axis displacements, a loudspeaker driven by a function generator was used to convey the sound to the sample. The frequency used was $1.3 \mathrm{kHz}$, with a sound pressure of $93 \mathrm{~dB}$ SPL (0.893 Pa), enough to induce out-of-plane displacements.

From that research a new mathematical scheme to measure the skin rigidity was proposed, where the anisotropic and heterogeneous nature of the skin is considered.

For the mathematical scheme, the medium was considered as a thin vibrating membrane where normal displacements to the surface are small enough, a dynamical equation was obtained in terms of partial derivatives as

$$
\begin{aligned}
\rho \frac{\partial^{2} u_{z}}{\partial t^{2}}= & c_{z x x z} \frac{\partial^{2} u_{z}}{\partial x^{2}}+c_{z y y z} \frac{\partial^{2} u_{z}}{\partial y^{2}}+2 c_{z x y z} \frac{\partial^{2} u_{z}}{\partial y \partial x} \\
& +c_{x} \frac{\partial u_{z}}{\partial x}+c_{y} \frac{\partial u_{z}}{\partial y}
\end{aligned}
$$

where $c_{z x x z}, c_{z y y z}$ and $c_{z x y z}$ are the rigidity moduli and the coefficients $c_{x}=\frac{\partial c_{z x x z}}{\partial x}+\frac{\partial c_{z x y z}}{\partial y}$ and $c_{y}=\frac{\partial c_{z x y z}}{\partial x}+\frac{\partial c_{z y y z}}{\partial y}$ are variations of $c_{z x x z}, c_{z y y z}$ and $c_{z x y z}$ with respect to the position $(x, y) . \rho$ is the density of the medium, $t$ is the time and $u_{z}(x, y)$ is the $z$ axis component of the displacement field that defines a surface whose amplitude at each point is time-dependent. By measuring $u_{z}(x, y)$ at five different times, a system of equations was constructed as
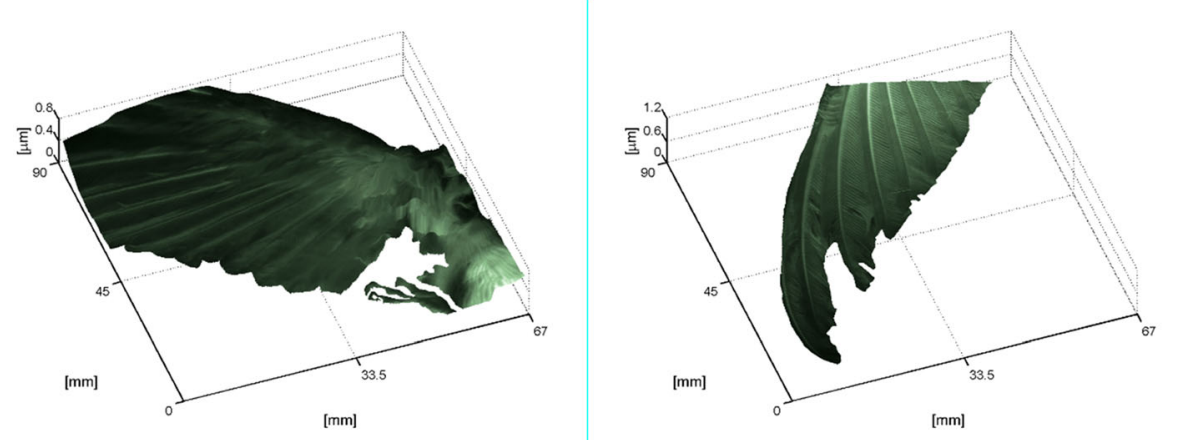

Fig. 7 Displacement map examples with bird's texture matching. Reprinted from [25]. Copyright [2013], SPIE 


$$
\left(\begin{array}{c}
\rho \partial_{t t}^{2} u_{z}^{(1)} \\
\vdots \\
\rho \partial_{t t}^{2} u_{z}^{(5)}
\end{array}\right)=\left(\begin{array}{cccc}
\partial_{x x}^{2} u_{z}^{(1)} & \partial_{y y}^{2} u_{z}^{(1)} & \ldots & \partial_{y} u_{z}^{(1)} \\
\vdots & \vdots & \ddots & \vdots \\
\partial_{x x}^{2} u_{z}^{(5)} & \partial_{y y}^{2} u_{z}^{(5)} & \ldots & \partial_{y} u_{z}^{(5)}
\end{array}\right)\left(\begin{array}{c}
c_{z x x z} \\
c_{z y y z} \\
\vdots \\
c_{y}
\end{array}\right)
$$

Then, the rigidity moduli $c_{z x x z}, c_{z y y z}$ and $c_{z x y z}$ can be found by solving the last system of equations.

The displacement fields normal to the surface under observation were obtained using DHI, where the sensitivity vector was chosen to be $\vec{k} \approx\left(0,0, k_{z}\right)$ in order to obtain $u_{z}(x, y)$. The evolution of the normal to the surface displacements obtained for different time periods of UV exposure are shown in Fig. 8. The right bottom graph shows the mean rigidity coefficients, $c_{z x x z}, c_{z y y z}$ and $c_{z x y z}$ vs time of UV exposure. The increment value of the rigidity coefficients means that the tissue elasticity was reduced after each period of UV radiation.

Other reports on vibration analysis of objects and biological membranes are worth of consideration [35-46]. The tympanic membrane's (TM) motion is very important in the hearing process, serving as a transformer of mechanical impedance between low impedance in the air in the ear channel, and the high impedance at the center of the eardrum and the umbo. As an example, dynamic excitation by acoustically induced motions on the tympanic membrane (TM) of a postmortem chinchilla using an ad-hoc DHI otoscope, is shown in Fig. 9. Here

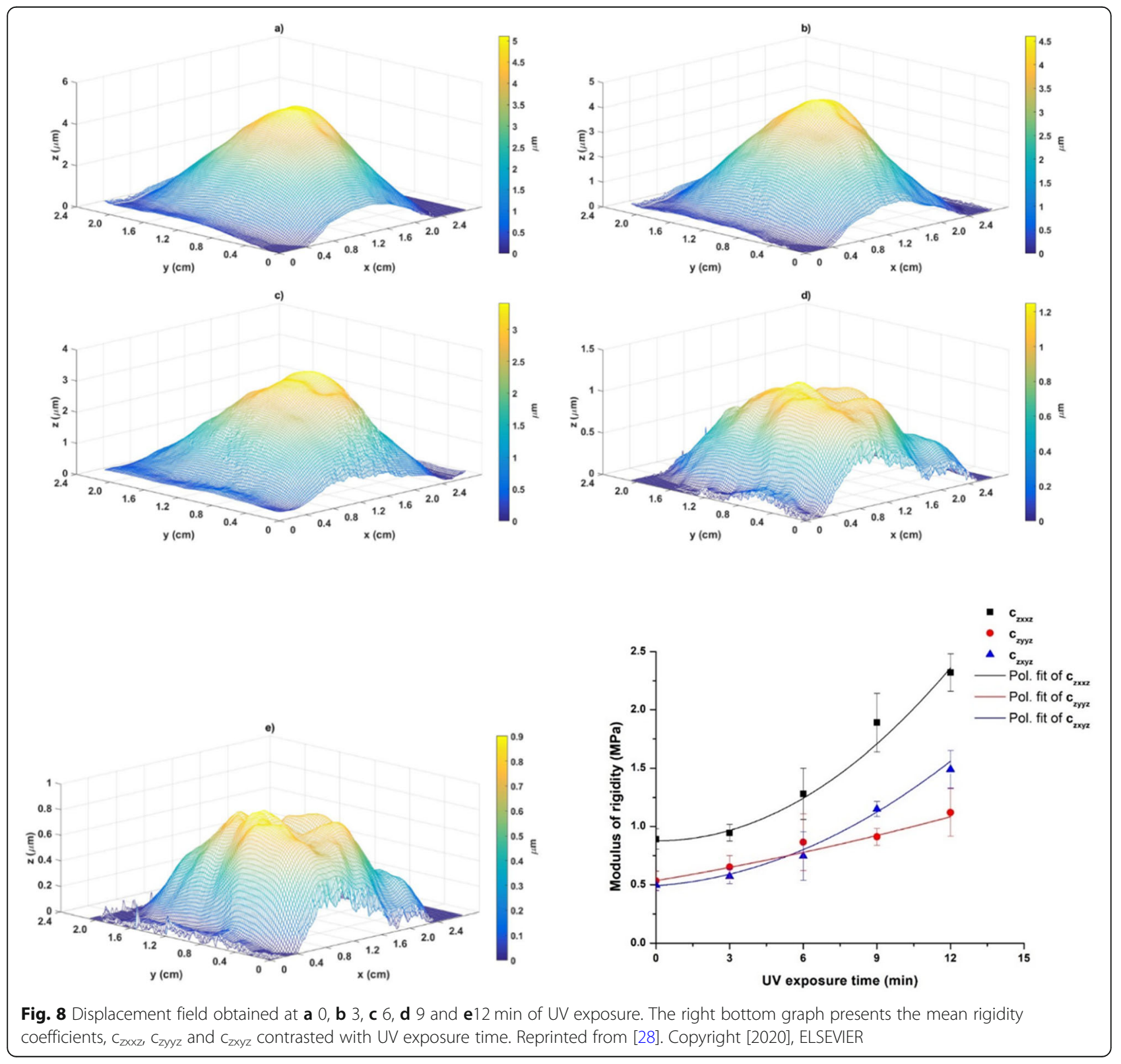




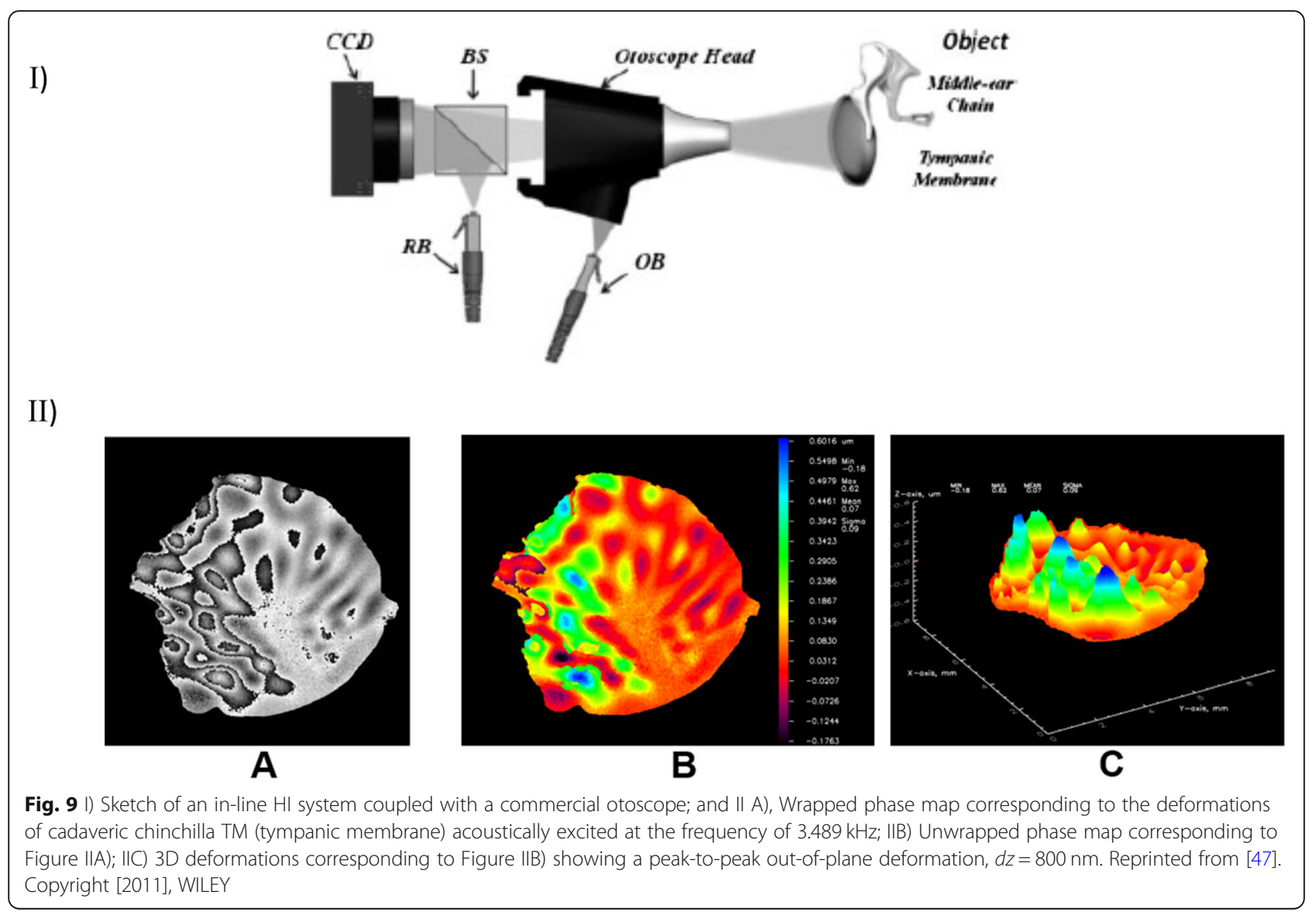

qualitative and quantitative deformation information of a TM subjected to controlled acoustic signals was reported, giving to the hearing specialists important information about the mechanical behavior of TM at different frequencies [47]. Other applications of DHI for the 3D reconstruction of mammal's TM, or the developing of robust algorithms to recover intensity images from holograms have been reported in Ref. [41, 48].

The sound-induced motions of the TMs, i.e., the deformation $u_{z}(x, y, t)$ normal to the TM's surface, is provoked by a continuous sinusoidal wave (acoustic) that can be represented by the following equation:

$$
u_{z}(x, y, t)=z_{m 0}(x, y, t) \sin \left[\varnothing_{m}(x, y, t)-\omega_{m} t\right]
$$

where $x$, and $y$, are the spatial coordinates, $t$ is the time, $z_{m 0}$ represents the amplitude of the acoustic signal, $\varnothing_{m}$ is the mechanical phase, and $\omega_{m}$ is the angular frequency $[49,50]$.

Consequently, DHI was applied for measuring soundinduced displacements, determination of the shape, and mechanical properties such as the strain on the TM. The TM surface height-change $h(x)$ may be found from the difference between the reconstructed phases which are recorded before $\phi_{1}$ and after $\phi_{2}$, the small tilt $(\Delta \theta)$ of the object illuminating beam by the following equation $[51,52]$ :

$$
\Delta \phi=2 k \sin \frac{\Delta \theta}{2}\left[x \cos \left(\theta+\frac{\Delta \theta}{2}\right)-h(x) \sin \theta+\frac{\Delta \theta}{2}\right]
$$

where $k=2 \pi / \lambda$, and $\theta$ is the object beam angle which is measured from the fixed position of object illumination to the axis perpendicular to the geometrical center of the CCD sensor. Therefore, the object height-change $h(x)$ can be determined from the difference between the reconstructed phases $\Delta \phi$ using equation (13), where $\Delta \phi$ was calculated with equation (7). Figure 10 shows the experimentally found shape of the tympanic membrane and a line profile of its surface.

In order to measure sound-induced displacements in the vibrating TM, the phase differences are calculated by capturing two holograms at two different points of the sound signal cycle, namely at $0^{\circ}$ (reference state) and $90^{\circ}$ (maximum amplitude of the 


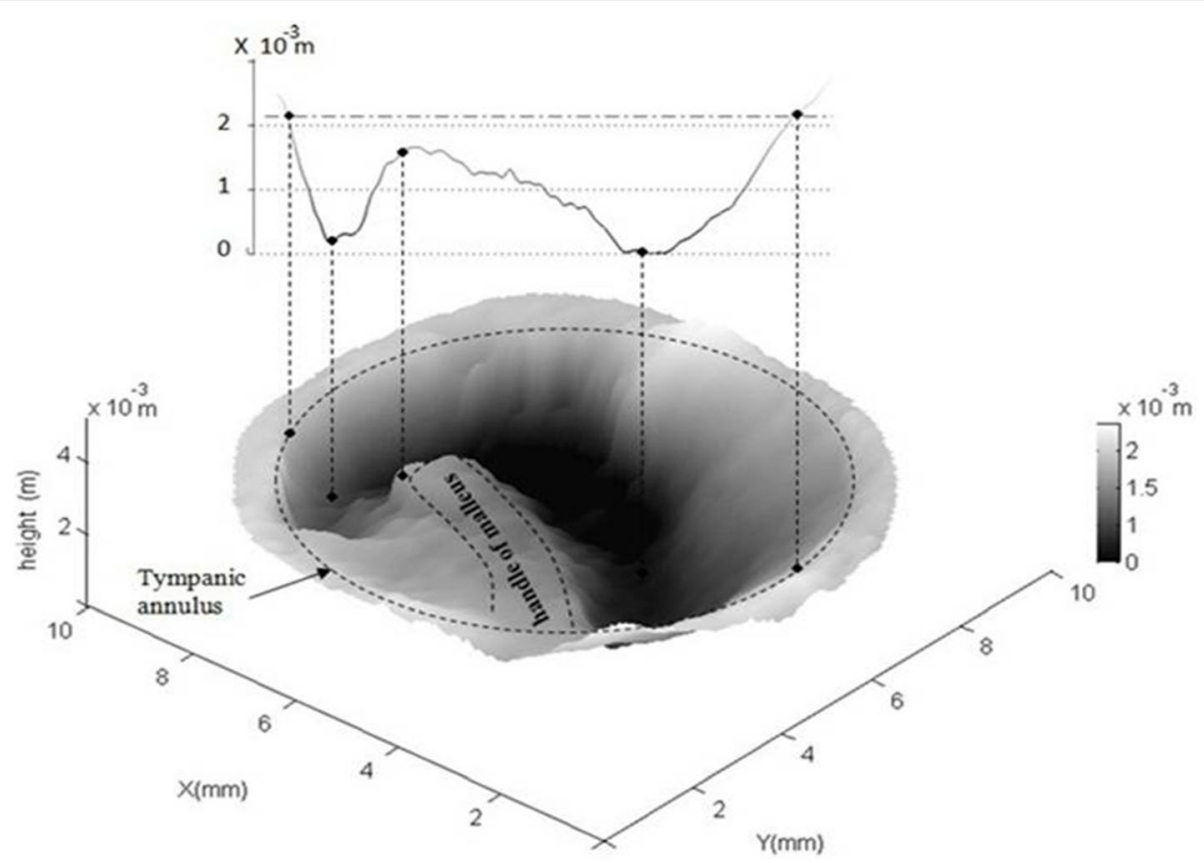

Fig. 10 3D reconstruction and surface profile of the TM. Reprinted from [51]. Copyright [2012], OSA

sound wave), i.e., two different instants of the soundinduced cycle. The synchronization of the signals (sound stimuli and external trigger to the CCD), is fundamental to secure and make the experiments repetitive, and with the exposure time short enough, $5 \mu \mathrm{s}$, to "freeze" the movement of the sample. Equation (9) is employed to find the displacements in the $\mathrm{z}$-direction.

Figure 11 shows the results of a cat's TM describing its motion as displacements over the entire surface. Here the sound frequencies were from $800 \mathrm{~Hz}$ to $8000 \mathrm{~Hz}$. Figure 11 a) shows the vibration patterns as wrapped optical phase, and b) illustrates the patterns like unwrapped phase maps.

Completeness studies of the tympanic membrane have been carried out using DHI in conjunction with a Confocal Laser Scanning Microscopy (CLSM): both were employed to measure the displacements and thickness of four cats' healthy TMs, and the study concluded on how the thickness (by region) of the eardrum affects the TM displacements when it is subjected to acoustic stimuli, in particular at 1.8, 5.2 and $12 \mathrm{kHz}$ [53]. The three specific regions studied were, R1 located in the PS posterosuperior quadrant of the eardrum; R2 in the AS anterosuperior quadrant between the umbo and the annulus; and R3 just below the end of the umbo near the annulus [54]. The displacements can be utilized to investigate the TM thickness changes without the need to invade the tissue. Figure 12 shows three "raw" fringe patterns (top), the resulting three wrapped phase maps (second row), and their corresponding unwrapped (third row) phase maps for one eardrum vibrating at 1.8, 5.2 and $12 \mathrm{kHz}$, respectively. The height displacements were calculated with equation (8). In order to determinate the TM's thickness with CLSM its Black Zen (Zeiss Efficient Navigation) analysis software was used. The 3D structure of the eardrum was reconstructed by stacking the confocal images in the $\mathrm{Z}$ direction. Figure 12 (bottom row) shows three CLSM orthogonal fluorescence images, where the white or bright "thin strip" of the image corresponds to the thickness of the membrane, showing that the TM was well delimited. In each image, the top of the fluorescent section corresponds to the outer surface of the TM and the bottom corresponds to its inner surface. The distance between these two surfaces was measured to quantify the thickness of the TM in each region [53].

DHI has been used to quantify mammal's vocal folds' dynamic displacements, see Fig. $13[55,56]$. This figure shows displacement maps in a pseudo 3D representation, the vibration pattern, and the wrapped phase map. There is a full 2D deformation in the vocal folds (VF) when an airflow passes through them.

These results exemplified the displacements variations along the VFs, showing the characteristic triangular form which is present during the opening of the VFs' vibrational stage. And the patterns indicate that the 


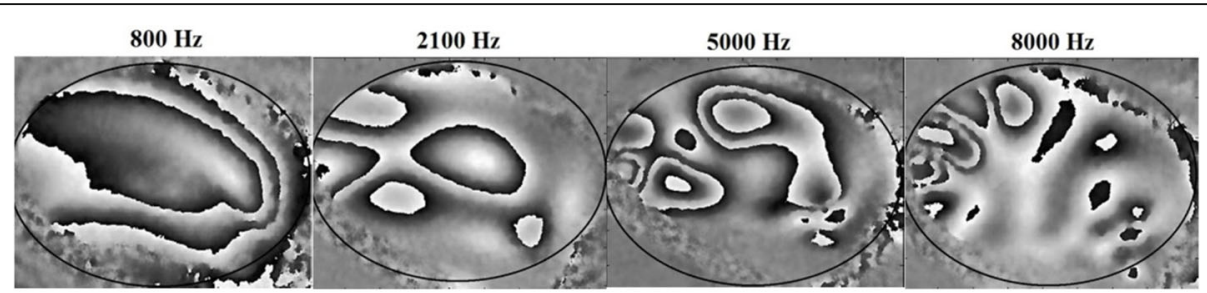

(a)
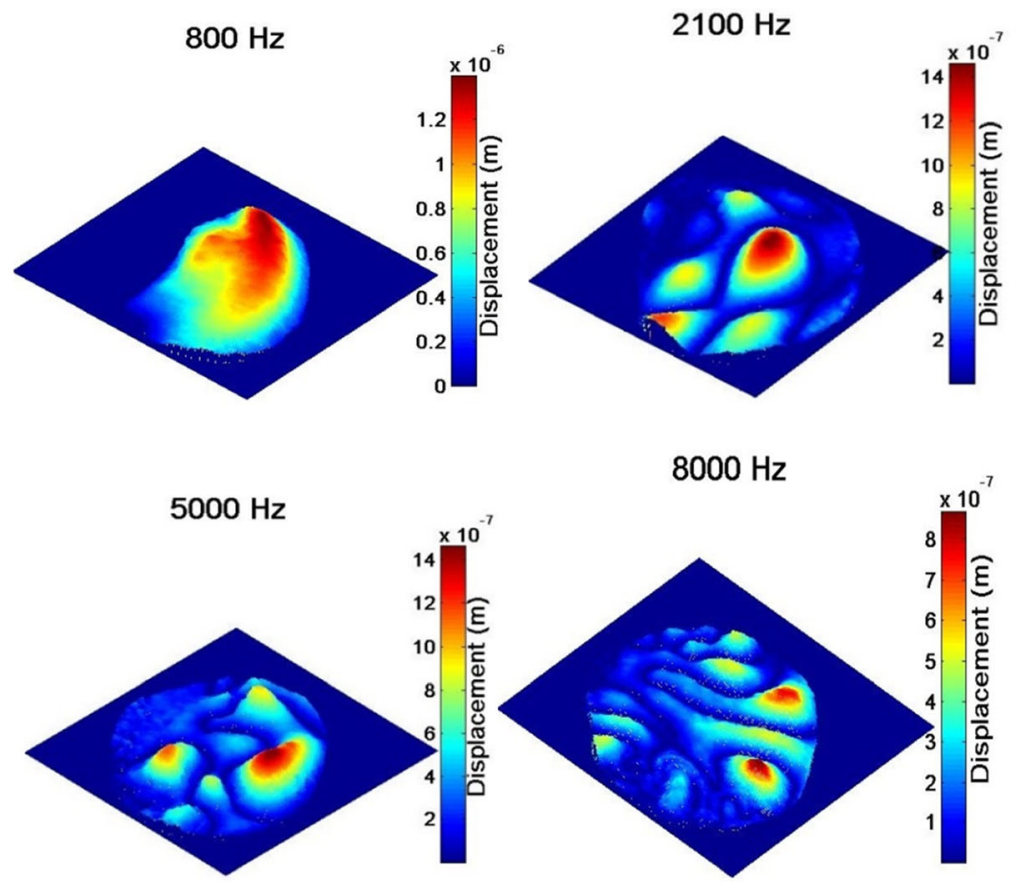

(b)

Fig. 11 Surface deformations in the z direction (a) Wrapped and (b) Unwrapped phase maps of the TM

movement propagates from the anterior to the posterior edges of the VFs. Complex behavior is observed for this tissue and not only showing deformation in one axis as expected.

Performing biomechanical tests is becoming a common task for DHI, mainly due to its advantageous fact of being able to retrieve the optical phase from a single hologram. In reference [57] a DHI using an endoscope retrieves the optical phase map from an oral cavity observing the tongue as it moves (see Fig. 14).

Here, it is possible to see the robustness of the retrieved signal in this complicated inspection showing almost no de-correlation in the interferometric signal. Some of this noise is present as this is a wet tissue with random specular reflections. However, even when the optical phase is affected, the use of high dynamic range cameras (more bits per pixel) helps to reduce this error $[58,59]$.

The flexibility in the DHI setups allows inspection of micrometric size structures such as cells. An example in reference [60] uses photosensitized HeLa cells that are studied when they interact with controlled laser irradiation. Figure 15 shows several images of the optical phase before and after these cells were irradiated. The tracking is presented for two particular cells (A and B) whose expression is highly reduced after $45 \mathrm{~min}$.

The biomechanics of another tissue that has been widely inspected is the bone from different species, such as: human [61], porcine [62] and bovine [63]. This is possible due to the fact that material engineering models are able to be applied to a wide range of experimental conditions regardless of the species. However, several of 


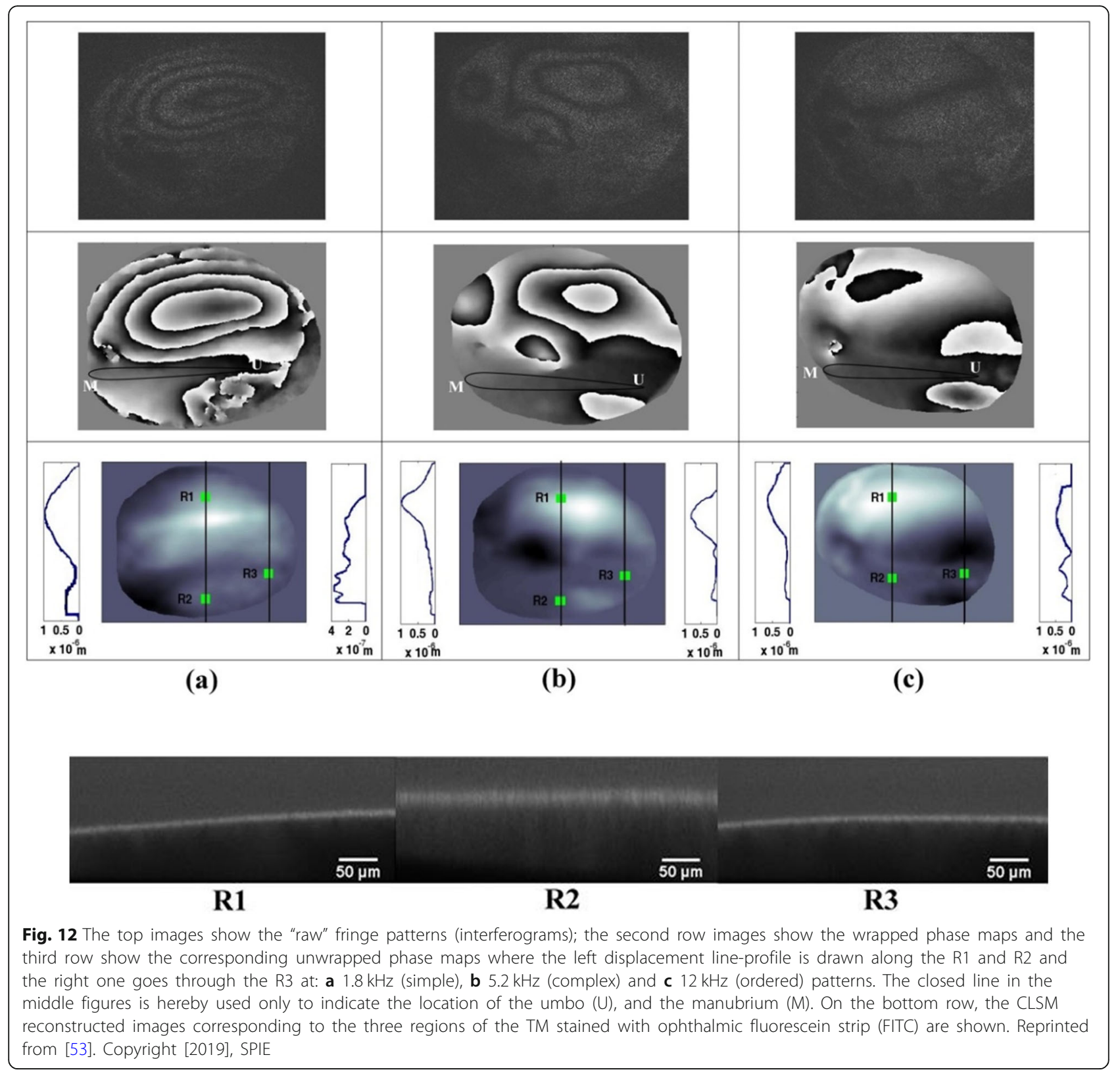

these investigations require a previous validation or a comparison with a numerical model. Sometimes, phantoms are used instead of a real tissue; to exemplify this point, in reference [64] a comparison (experimental vs theoretical measurements) is presented in an artificial tooth. The aim of this work is to study the deformation of a tooth due to the mastication forces. Figure 16 shows two fringe patterns during the deformation test.

This experimental deformation data is compared with that coming from a finite element model (FEM) simulation of the same experiment. The FEM results are shown in Fig. 17 where it is possible to observe a good correlation between the experimental and the simulated results. This correlation is one of the advantages that makes DHI so widely applied in different conditions and with different samples under study.

Bone analyzes using DHI are focused on its mechanical strength and how external factors could modify this. Reference [65] presents a study where the mechanical response of a porcine femoral bone is analyzed when it suffers a compression. In this study the bones are affected by means of cortical hole drillings which simulate the condition when a prosthetic plaque needs to be fixed in order to heal a fractured bone. A 

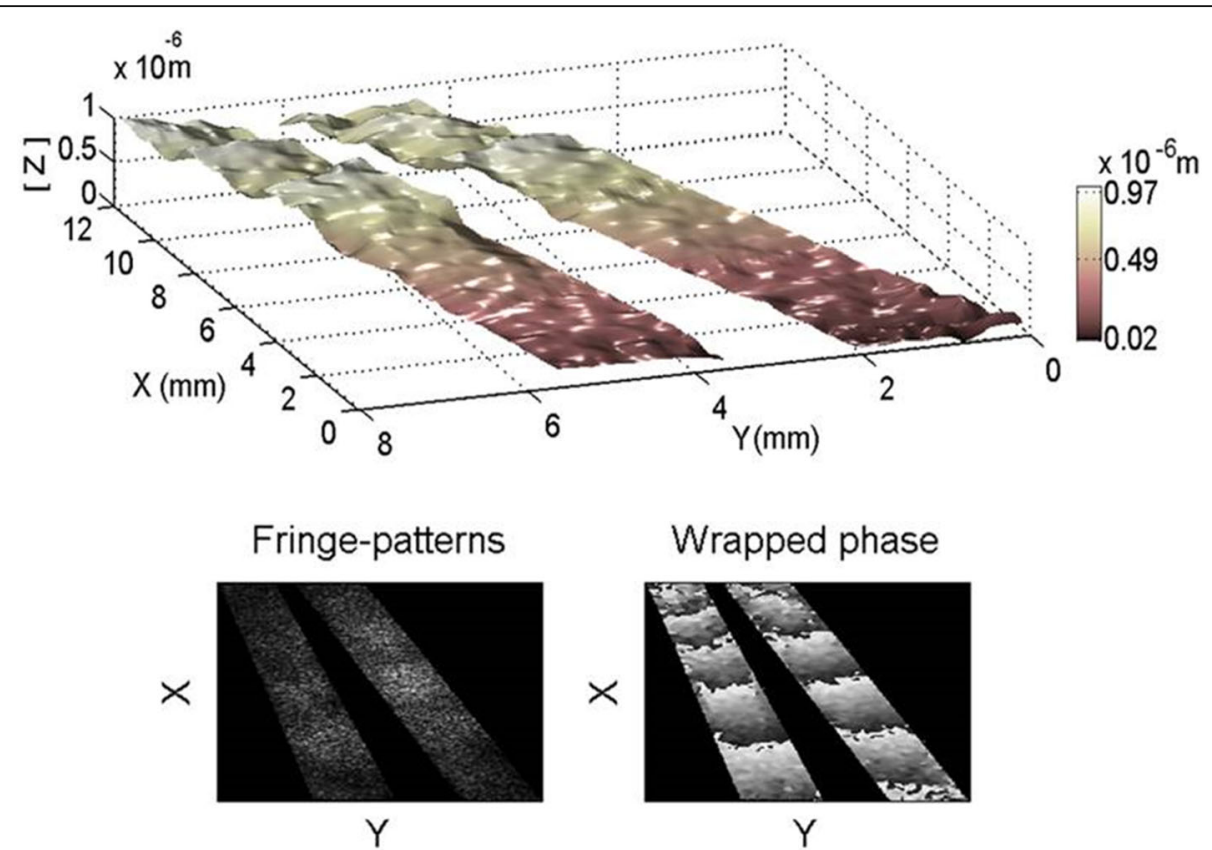

Fig. 13 The top image is the VF unwrapped vibrating map. The bottom ones refer to the fringe pattern (left) and its corresponding wrapped phase (right). Reprinted from [56]. Copyright [2016], IOP

continuous compression load is applied to several bones with different conditions. Some have 2, 4 or 6 cortical holes $(6.5 \mathrm{~mm}$ of diameter) and their mechanical deformation is compared with the fourth set of non-drilled bones (control group). The study presents the displacement maps at some selected compression values for comparison purposes. Figure 18 shows the results for three different non-drilled bones (control response) while Fig. 19 shows the average response of the drilled bones when the compression reaches 400 lbs. Comparing these two sets of images it is possible to observe how the anisotropic response of a healthy bone is affected in the presence of a cortical hole. Furthermore, this mechanical modification increases as the number of the hole number increases. Cortical holes remove bone material, weakening it and making a fracture more likely to happen.

A further study in affected bones was proposed in reference [66], where specimen samples from bovine cortical bones are tested. Here, demineralization $(d m)$ and dehydration $(-\mathrm{H} 2 \mathrm{O})$ are the affectations applied to a group of specimens. Resulting in four groups as Fig. 20 indicates. The controlled compression required the design of an ad-hoc micro compression testing machine (MCTM) able to apply the controlled deformation during the test. The experimental set up is observed in Fig. 21.

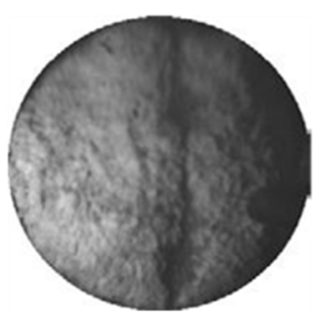

(a)

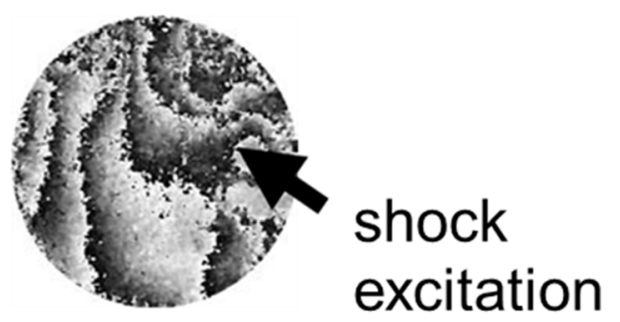

(b)

Fig. 14 In vivo investigation inside the oral cavity, (a) image of the investigated part (tongue), (b) phase map corresponding to the deformation produced by mechanical excitation of the tongue. Reprinted from [56]. Copyright [2005], SPIE 


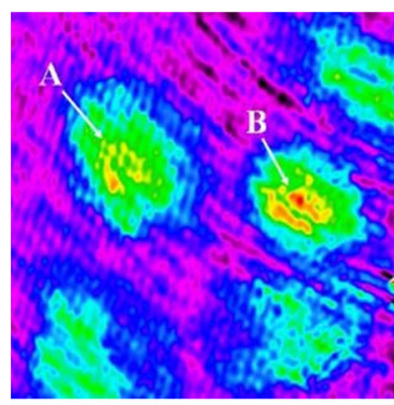

(a)

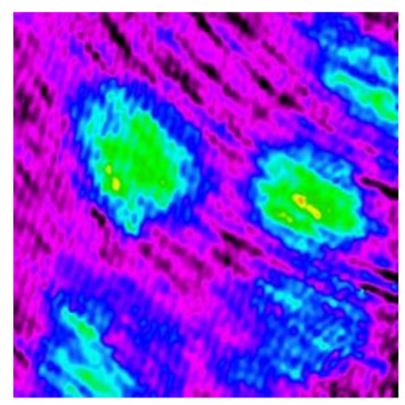

(b)

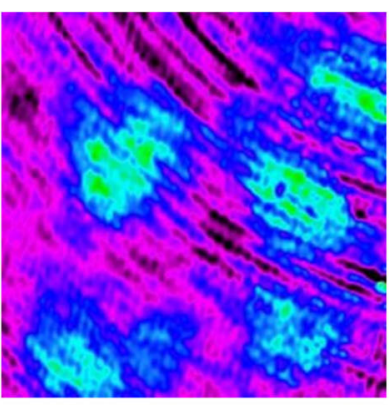

(c)

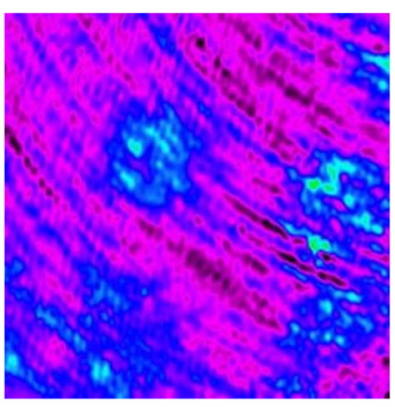

(d)

Fig. 15 Distribution of phase retardation in the sample before the irradiation (a), right after 60-s irradiation (b), in 45 min (c) and in $40 \mathrm{~h}$ (d) after the irradiation. Reprinted from [60]. Copyright [2016], OSA

After processing all the bones' responses when they are compressed, an average displacement map is retrieved for each case. Figure 22 shows the comparison between the control group $(\mathrm{m}+\mathrm{H} 2 \mathrm{O})$ and the one demineralized but hydrated (dm@4 h+ H2O) at three different compression values. According to this information, there is not a big mechanical difference between these two groups as the demineralized bones still act as a healthy one in this compression range.

Figure 23 shows the comparison between the dehydrated but mineralized bones (m-H2O@48 h) and the demineralized and dehydrated ones (dm@4 h-H2O@48 h). Here, it is possible to see a remarkable difference between the control group and the dehydrated but mineralized bones that are highly affected even when their mineral is still present. This difference implies a bigger deformation and the fracture value is decreased, making them more prone to be fractured. The last case where both components are affected has a plastic response indicating an extreme strength affectation of the sample. Analyzing this information it is possible to see that dehydration affects more bone strength than the demineralization.

All of the above has shown that the use of DHI has been extended beyond the traditional study of mechanical properties of materials to biomechanical properties of biological objects, involving a diversity of disciplines like mechanics, biology, chemistry, and tissue engineering among many others.

\section{Digital holographic microscopy (DHM)}

In the micrometric size inspection, new configurations have been developed in order to simplify the alignment and measurement of these samples. Reference [67] presents a novel transmission DHI set up able to identify micrometric size particles $<50 \mu \mathrm{m}$. The optical phase is able to reconstruct pollen particles as

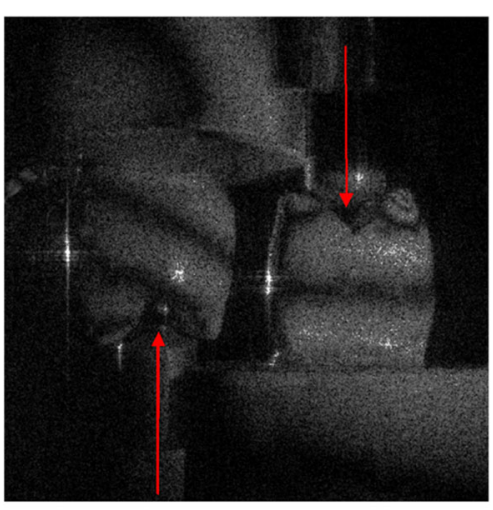

(a)

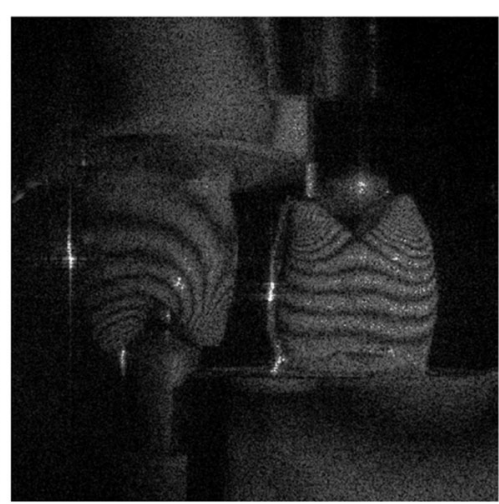

(b)

Fig. 16 Interferograms of an artificial tooth under (a) $100 \mathrm{~N}$ and (b) $250 \mathrm{~N}$ loads applied by a brass tool. The red arrows indicate the load directions. Due to the high dynamic range of the resulting image, the intensity scale is logarithmic. Reprinted from [64]. Copyright [2014], SPIE 


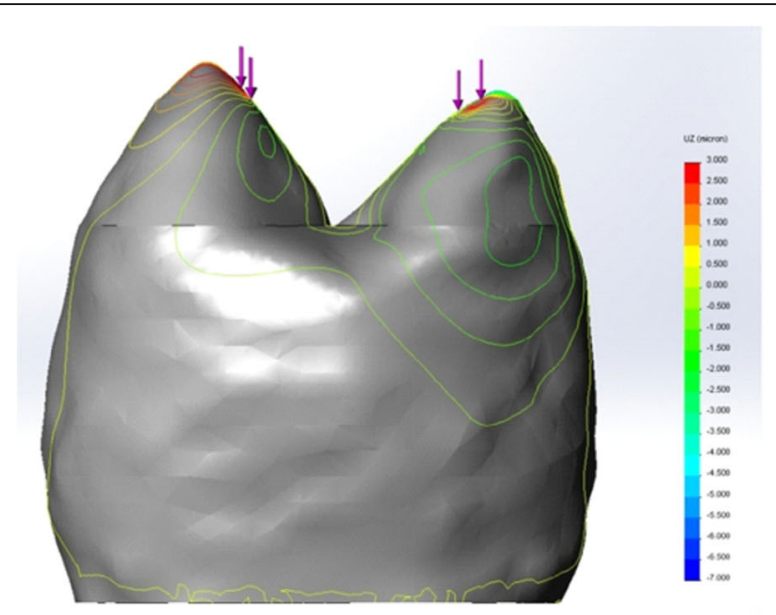

(a)

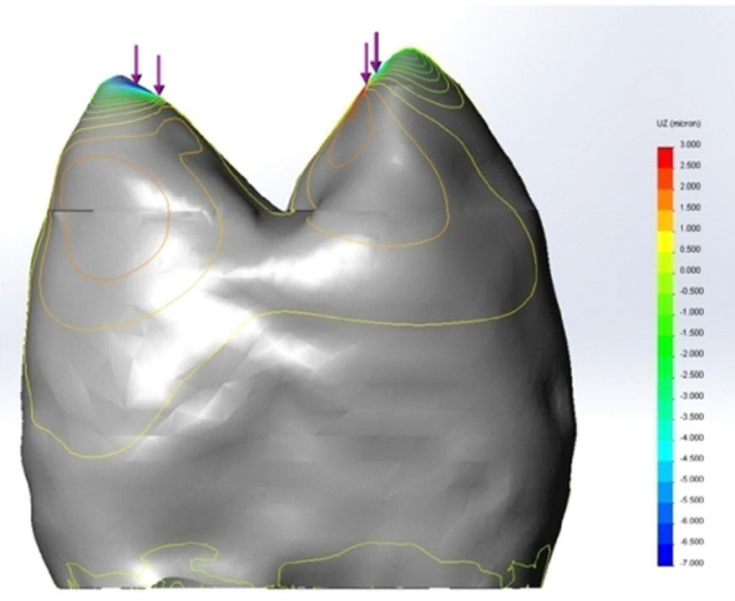

(b)

Fig. 17 Simulated deformation of an artificial tooth, calculated using FEM: a front side, $\mathbf{b}$ backside. Reprinted from [64]. Copyright [2014], SPIE

Fig. 24a shows, while Fig. 24b and c show the optical phase for a calibration step used to validate the biological measurements. Fig. 25 compares the image retrieved with confocal microscopy with the one from transmission DHI for the same pollen particles. It is notorious the advantage of DHI in transmission (using the simplified microscopy set up in reference [67], which renders the microscopic image of the particles and retrieves their profile.

When a phase object is illuminated its transparency or translucent nature does not provoke a large amplitude variation of the transmitted wavefront, which is not the case for phase changes. In these applications, the optical path difference is a consequence of a change in the refractive index distribution along the optical path through the sample. Also, physical thickness measurements are possible. These measurements represent a paramount aspect of DHM and are covered by the quantitative phase microscopy (QPM). These techniques allow the quantification of optical thickness and refractive index changes with nanometric accuracy based in the Fourier phase microscopy (FPM) method. One of the first reports in this field was carried out by Popescu et al., in 2004 [68]. A typical DHI configuration to measure the refractive index change of a phase object is based on an off-axis setup. The general expression [68] for the spatially resolved quantitative-phase images in, e. g., a cell sample, is given by:

$$
\phi(x, y)=\left(\frac{2 \pi}{\lambda}\right) \int_{0}^{h(x, y)}\left[n_{C}^{z}(x, y, z)-n_{0}\right] d z
$$

where $\lambda$ is the illumination wavelength, $h$ is the local thickness of the cell, and $n_{0}$ is the refractive index of the surrounding liquid; the symbol $n_{C}^{z}$ represents the refractive index of the cellular material, mostly an inhomogeneous function of $x, y, z$. Then, quantitative measurements of refractive index distributions on phase objects can be calculated. Measurements of changes in the refraction index in microscopic transparent or semi-transparent samples by DHI have been addressed using interference microscopy techniques such as the Mirau or Linnik interference microscopes. These are based on spatial carrier fringe-pattern analysis, and their developments are a version of the classical Zernike and Normarski optical phasecontrast microscopy [69].

A very important manuscript showing advances in biological applications based on DHM, was reported by Marquet et al. in 2005 [69]. The publication of this paper and others published by the Depeursinge group from Ecole Polytechnique Federale de Lausanne, are taken by many authors as introductory in the use of DHM in biological field [68, 70], with the original demonstration of nanometric surface profiling and have carried out quantitative cellular imaging and characterization studies. Popescu refers those papers as QPI-based techniques by DHM used for imaging live cells [68]. As an example of DH-QPM imaging, Marquet et al [69]. show in Fig. 26 the image of living mouse cortical neurons and their reaction to a hypotonic shock.

Several biological samples have been studied using DHI microscopy that follow the latter publication's ideas and results, take for instance some recent applications of DHM to study bio-samples. In Fig. 27 Bianco et al [71]. report a hybrid system of an optical tweezer coupled to DHM to quantify the bio-volume of red blood cells (RBCs). 


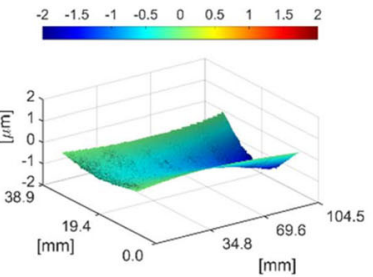

(a)

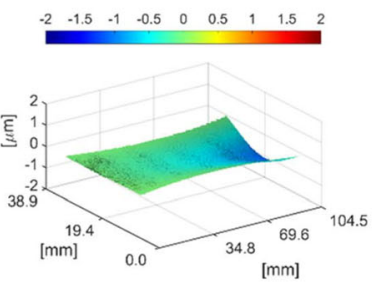

(d)

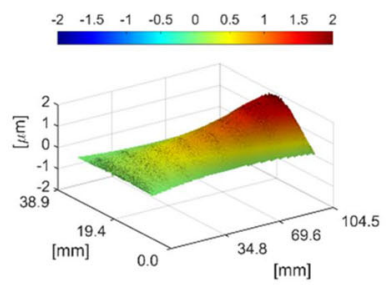

(g)

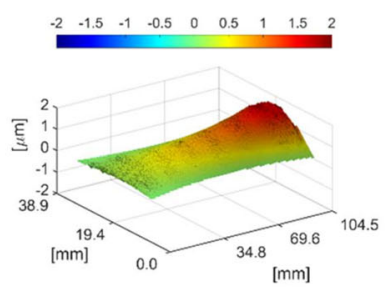

(j)

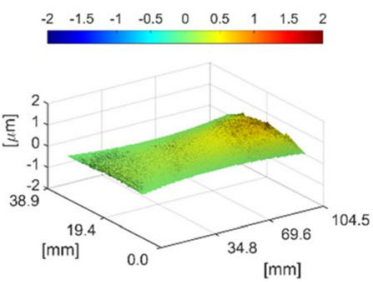

(m)

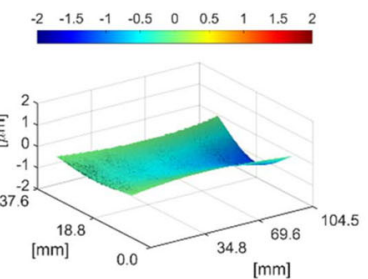

(b)

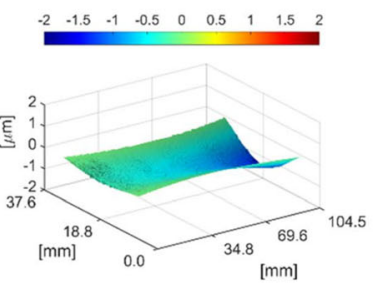

(e)

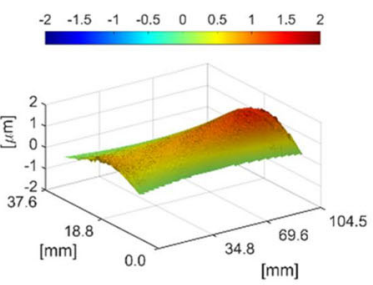

(h)

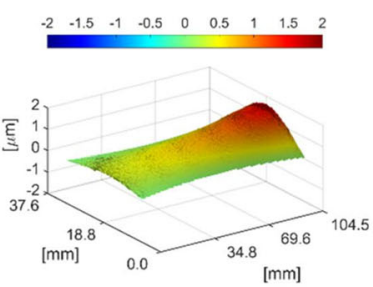

(k)

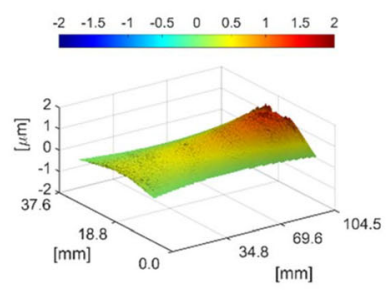

(n)

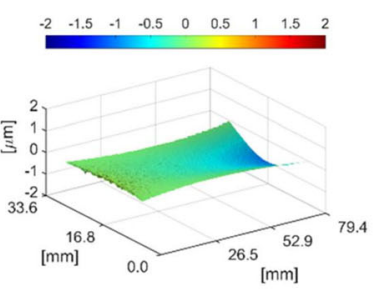

(c)

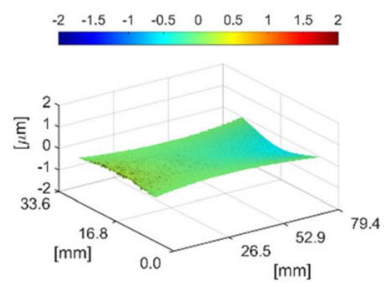

(f)

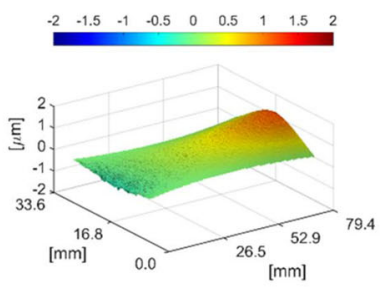

(i)

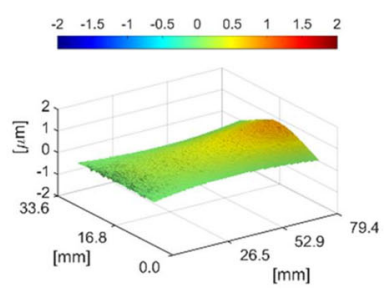

(I)

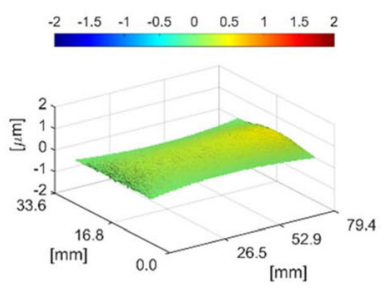

(0)

Fig. 18 Displacement map comparison for three different non-drilled bones. Each column represents a different bone, and each row reflects the compression value of interest at (a)-(c) 30 lbs., (d)-(f) 50 lbs., (g)-(i) 100 lbs., (j)-(I) 200 lbs., and (m)-(o) 400 lbs. Reprinted from [65]. Copyright [2017], OSA

Rastogi et al [72]. calculated the thickness and reconstructed the 2D phase profile of a $E$. coli bacteria, shown in Fig. 28. Barroso et al [73], .present a multispectral quantitative phase imaging system with DHM to characterize a dissected murine retina by label-free refractive index measurement, results seen in Fig. 29. The wavelength is tuned from 800 to $850 \mathrm{~nm}$ at steps of $10 \mathrm{~nm}$.
Ref. [74] presents a very clever combination of technology involving a smartphone, a DVD optical head and DHM algorithms to perform imaging of cells: the results are in Fig. 30, where the reconstructed image from red blood cells, HeLa cells and a micro channel on an aluminum coated silicon substrate, are shown. 


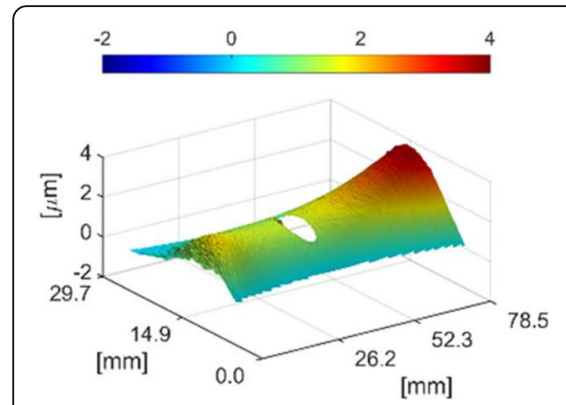

(a)

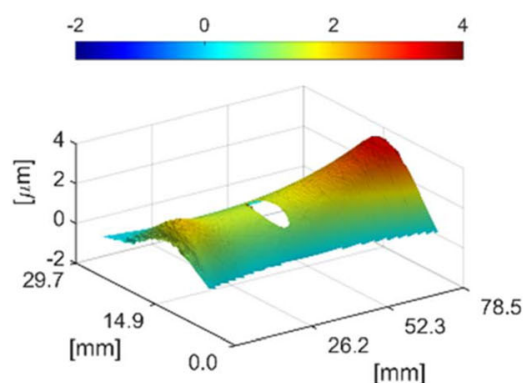

(d)

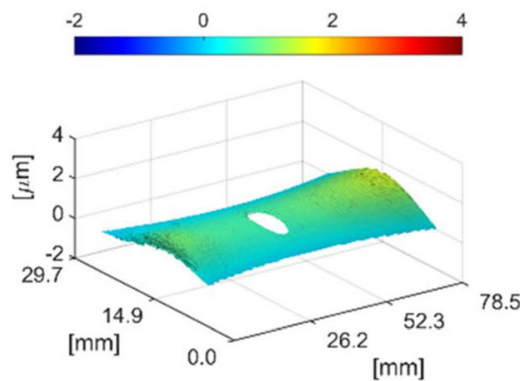

(g)

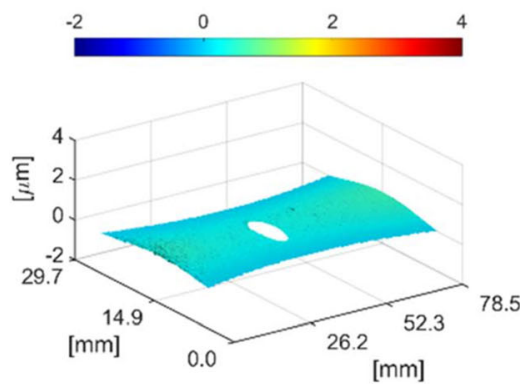

(j)

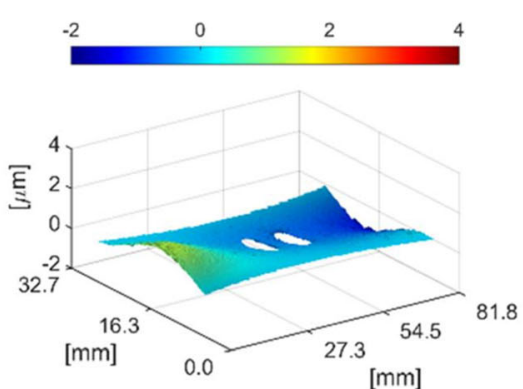

(b)

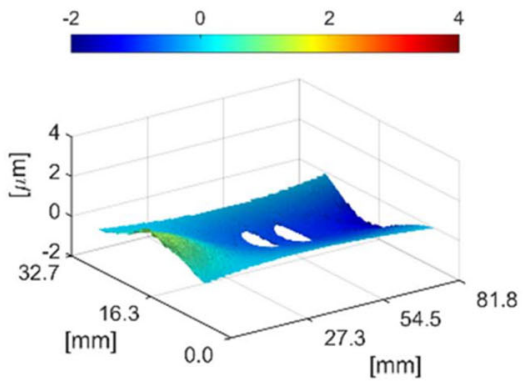

(e)

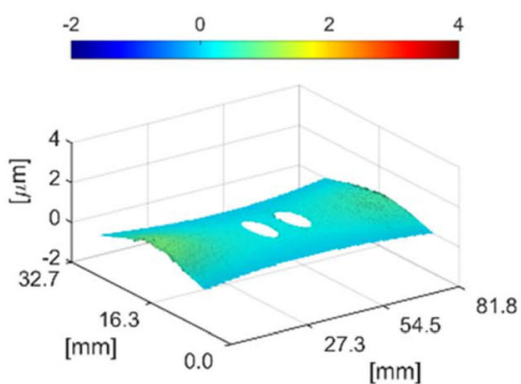

(h)

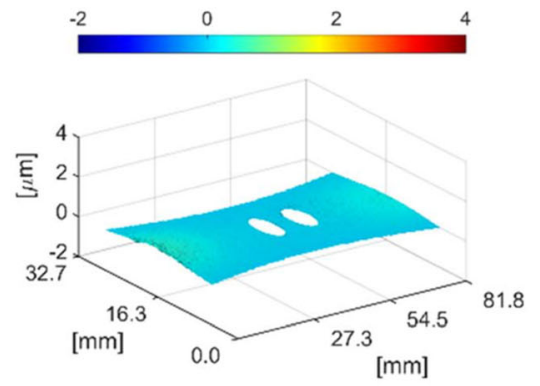

(k)

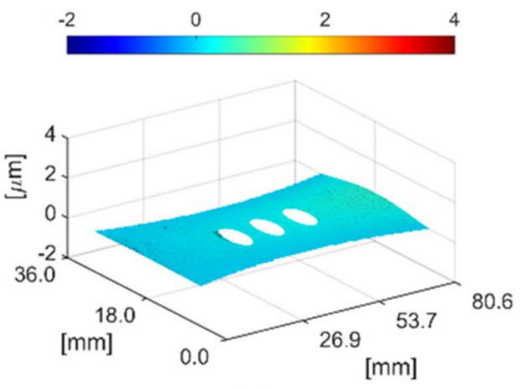

(c)

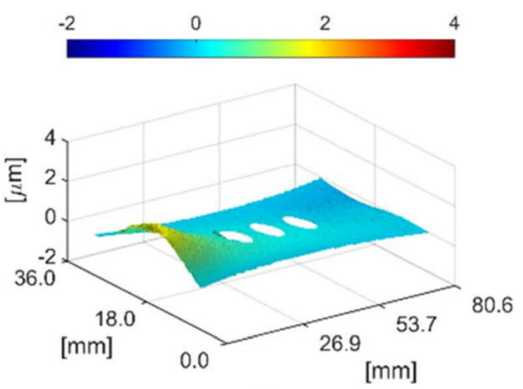

(f)

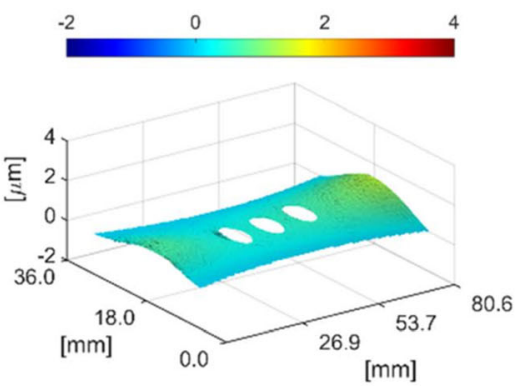

(i)

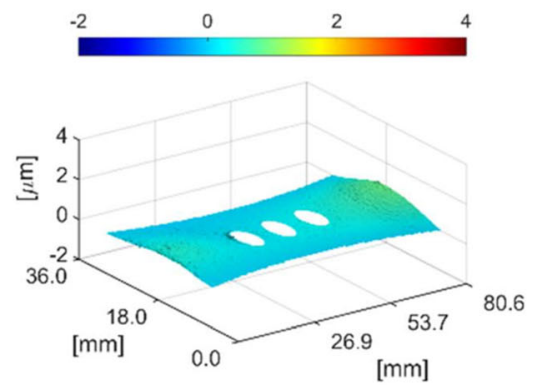

(I)

Fig. 19 Retrieved displacement maps for three bones with 2, 4, and 6 cortical holes at (a)-(c) 50 lbs., (d)-(f) 100 lbs., (g)-(i) 200 lbs., and (j)-(I) 400 lbs. with a $6.5 \mathrm{~mm}$ diameter drill bit. Reprinted from [65]. Copyright [2017], OSA

Finally, by using partially spatially coherent light with DHM, Dubey et al [75], showed how the sperm cell motility is affected when exposed to different levels of oxidative stress, see Fig. 31. Many of these techniques including offaxis, phase-shifting and common-path methods, are compiled in several textbooks such as those published by Popescu and Kim $[68,70]$ and indeed many other authors.

\section{Biomedical contributions from electron holography}

Brief background on electron holographic interferometry At the time that Gabor published [76, 77] his work on Electron Holography the proposed setup consisted of an in-line, or on-axis, wavefront combination, namely, the so called object and reference beams 


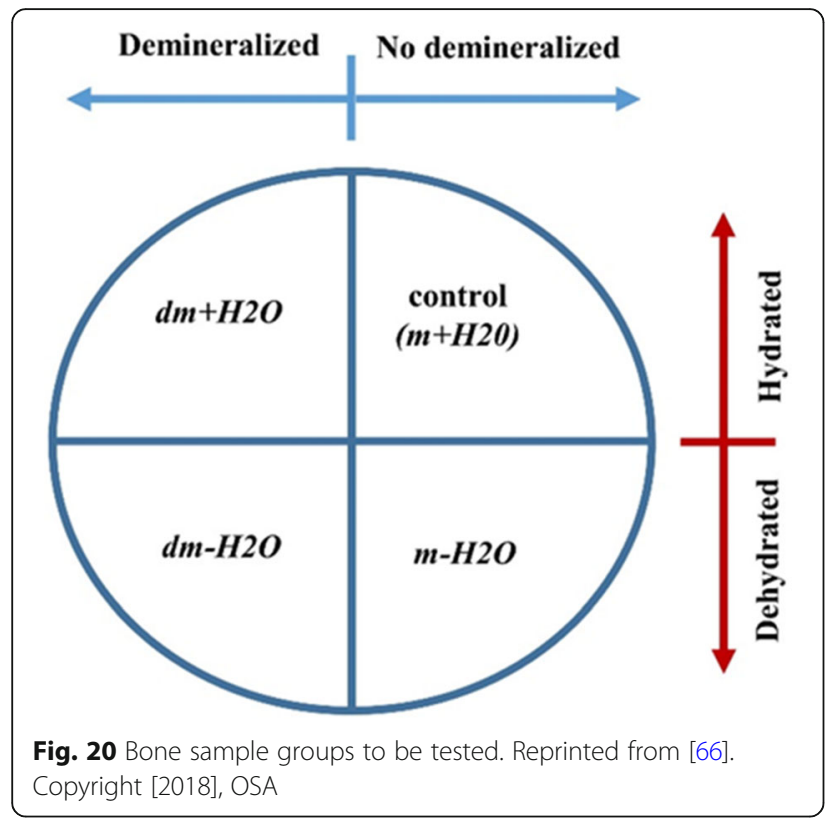

originated from the same "optical" axis, which is the axis where observation of the object was made. This in itself imposed a rather dramatic drawback in the technique since the reference and object electron beams appeared in-line on the sensor used to collect the electrons, that is, the high intensity (proportional to the electron beam irradiance) of the reference beam overshadowed the object, making it almost impossible to observe and thus recover the object information. The object wavefront reconstruction used an all optical setup that consisted in illuminating the hologram recorded on the film with a coherent monochromatic beam that worked in the visible part of the electromagnetic spectrum. This monochromatic beam was later replaced by the laser which meant that a brighter and more coherent light beam could be used to reconstruct the object wavefront imbedded in the randomness structure of the interferogram recorded in the hologram. So, on one hand, the lack of a reliable and coherent source of electrons and on the other the fact that holograms did not always result upon reconstruction in a well-defined object image (and indeed sometimes no object image was recorded at all), meant that Electron Holography was not practiced consistently in the Microcopy world. In the electron microscopes of that time, the reference and object beams were sometimes combined by using the concept of the Mollenstedt type electron biprism [78] which is a device that works as an optical Fresnel biprism, i.e., it serves to divide equally a beam into two wavefronts, also called amplitude division interferometers.

Electron Holography in microscopy was benefited by both the appearance of the CCD that replaced the wet chemical process, and also by the introduction of the field-emission electron gun in the microscope since it provides a high power and coherent electron beam which is a must for the development of Electron Holographic Interferometry (EHI) [79-81]. The technique invented by Gabor was devised to seek a reliable way to correct the aberrations inherent in electron microscopes, mainly the spherical aberration which meant that the observed images of angstrom size were defocused. But, the developments in Digital Holographic Interferometry can all be applied to EHI, so the latter has taken a major relevance in microscopy in the last few decades, and recently has shown the potential to be a tool that is able to extract experimental "real" information that may serve as the

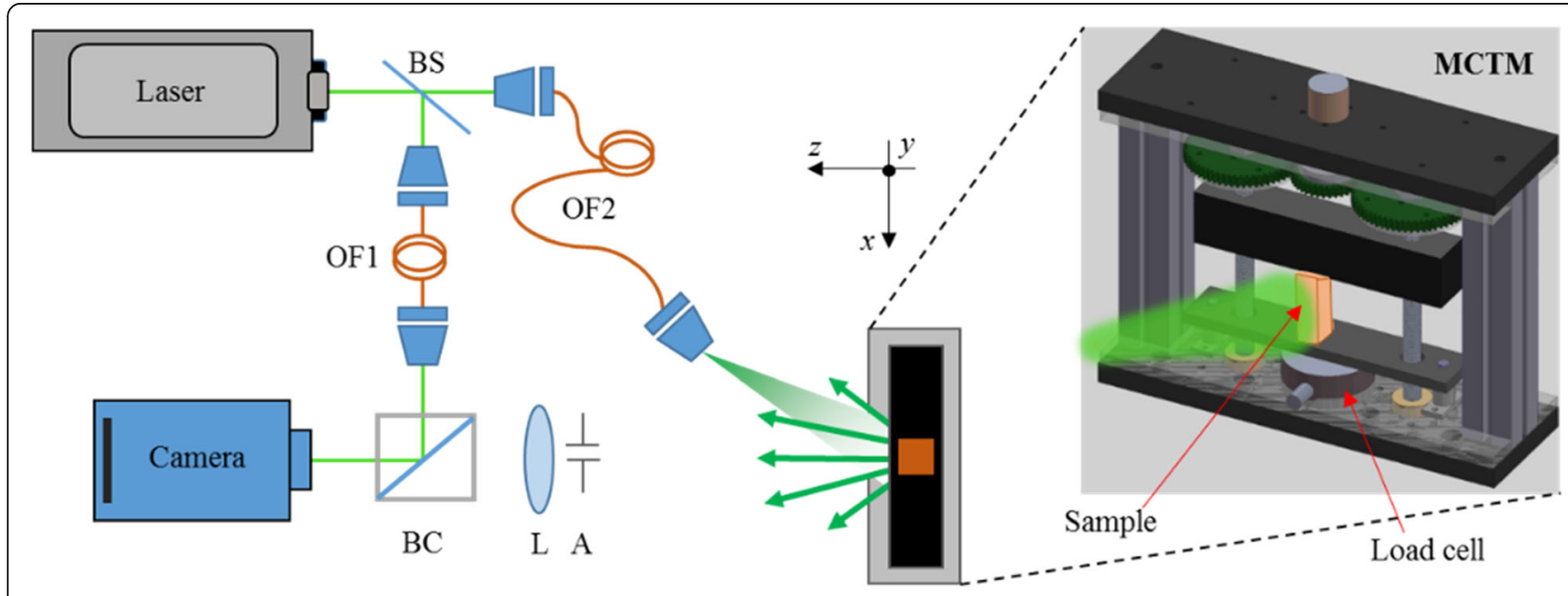

Fig. $21 \mathrm{DH}$ set up for the cortical bone compression tests. Reprinted from [66]. Copyright [2018], OSA 


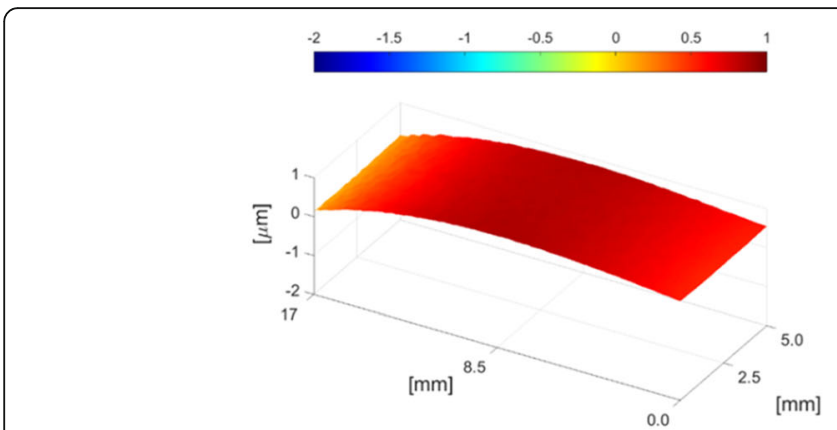

(a)

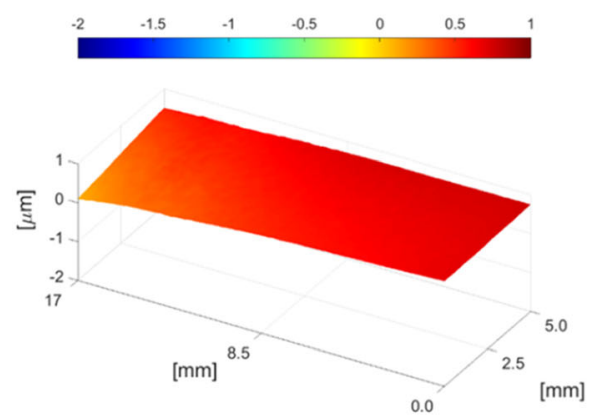

(c)

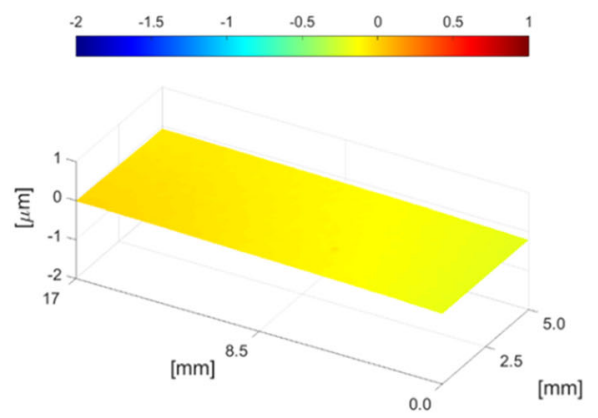

(e)

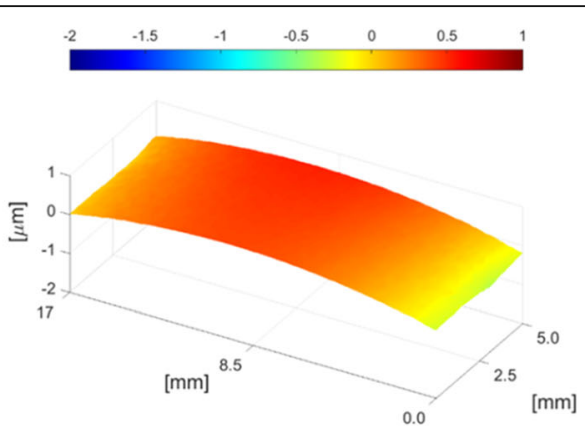

(b)

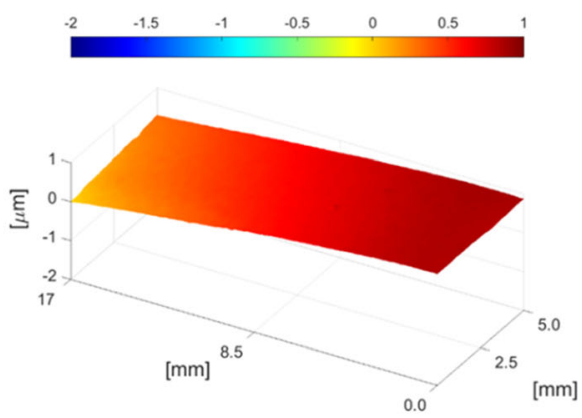

(d)

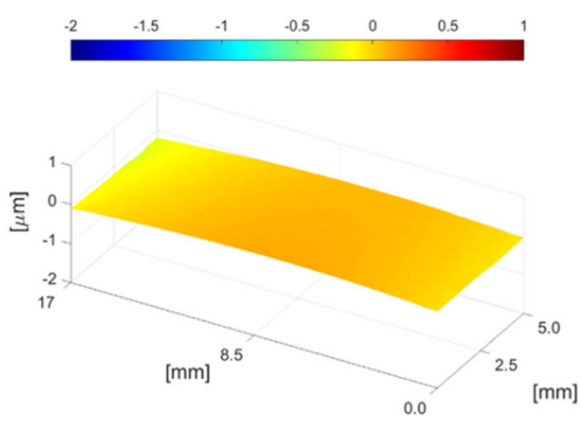

(f)

Fig. 22 Average surface displacement map comparison between $m+\mathrm{H} 2 \mathrm{O}$ and $\mathrm{dm} @ 4 \mathrm{~h}+\mathrm{H} 2 \mathrm{O}$ left and right column respectively, for (a, b) 100 (c, d) 200 and (e, f) 300 lbs. Reprinted from [66]. Copyright [2018], OSA

basis for the correction of theoretical descriptions, both product of non-proved or empirically formulated theories in Nano Sciences and Technology.

\section{Off-Axis electron holographic interferometry}

In Electron Holographic Interferometry (EHI) the intensity distribution at one point $r=(x, y)$ may be expressed as,

$$
\begin{aligned}
\mathrm{I}(\boldsymbol{r})= & \mathrm{I}_{\mathrm{R}}(\boldsymbol{r})+\mathrm{I}_{\mathrm{O}}(\boldsymbol{r})+\mathrm{I}_{\text {inel }}(\boldsymbol{r}) \\
& +2 \mu a(\boldsymbol{r}) b(\boldsymbol{r}) \cos \left[2 \pi \Delta \boldsymbol{k} \boldsymbol{r}+\Delta \phi(\boldsymbol{r})+\phi_{0}(\boldsymbol{r})\right]
\end{aligned}
$$

where $\mathrm{I}(\boldsymbol{r})$ is the intensity distribution on the image plane as recorded by the camera's sensor. $\mathrm{I}_{\mathrm{R}}(\boldsymbol{r})$ and $\mathrm{I}_{\mathrm{O}}(\boldsymbol{r})$ are the intensities of the reference and the object wave respectively. $\Delta \phi(r)$ represents the phasedifference between the reference and the object waves (see eq. 8), $\phi_{O}(\boldsymbol{r})$ represents a phase term that describes the position of the fringe pattern with respect to the camera (also known as lateral phase and/or fringe carrier) where the dependence on $r$ can e.g., describe distortions from the camera (i.e., the fiber optic bundles for electron microscopy type cameras). $\mathrm{I}_{\text {inel }}$ represents incoherent contributions coming from inelastically scattered electrons or stray emission from 


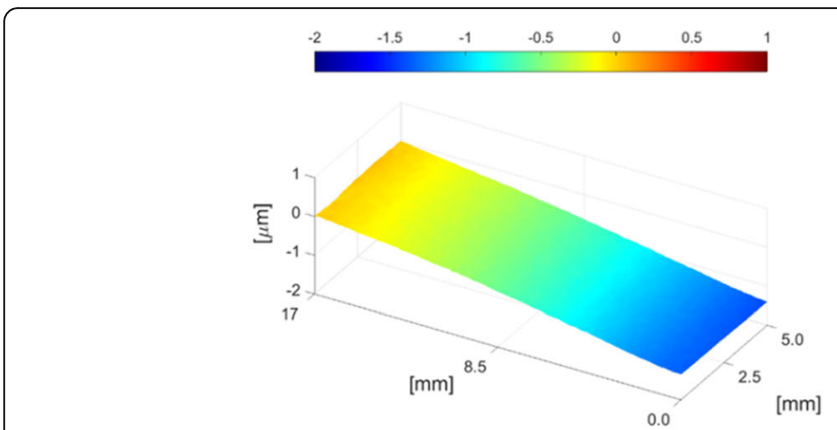

(a)

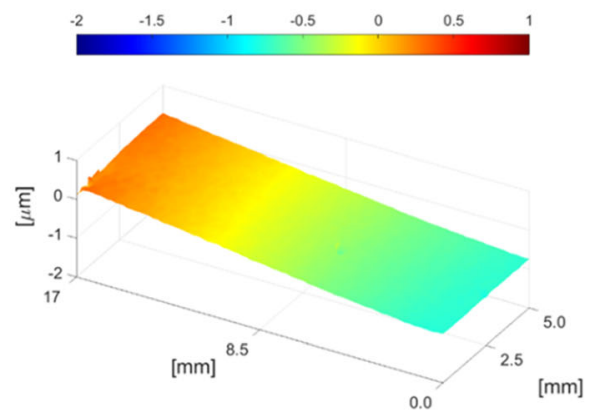

(c)

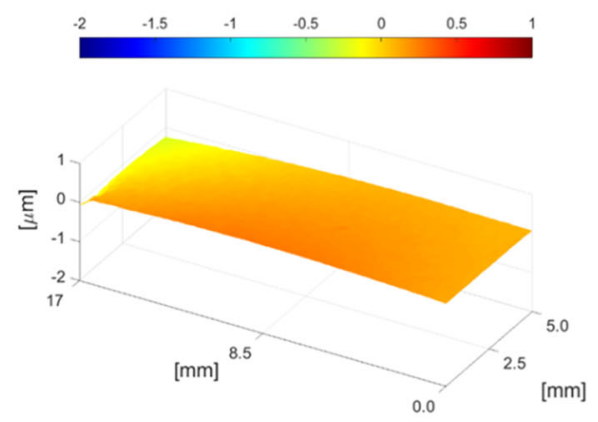

(e)

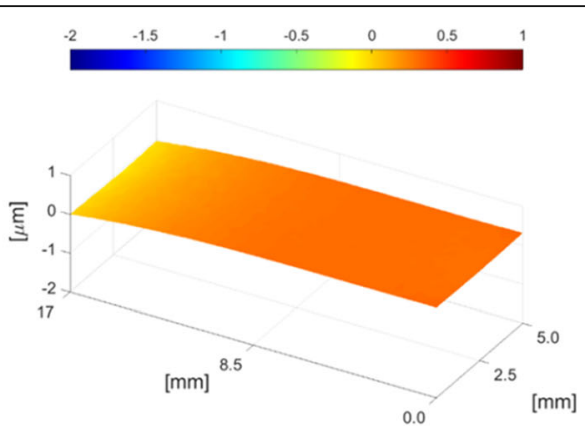

(b)

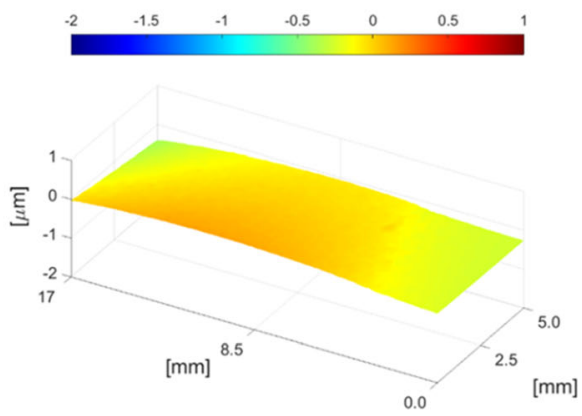

(d)

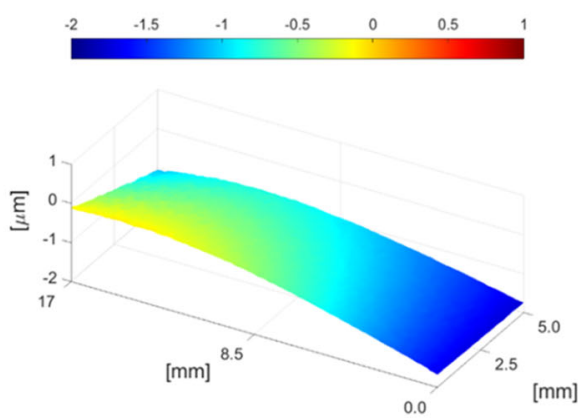

(f)

Fig. 23 Average surface displacement map comparison between m-H2O@48 h and dm@4 h-H2O@48 h left and right column respectively, for (a, b) 100 (c, d) 200 and (e, f) 300 lbs. Reprinted from [66]. Copyright [2018], OSA

a different source to the hologram, and $\mu$ is the contrast of the interference fringes. This contrast is preferably measured without an object for transmission type holography and with a perfect mirror for reflective holography, and it is given by,

$$
\mu=\frac{\left(I_{\max }-I_{\min }\right)}{\left(I_{\max }+I_{\min }\right)}
$$

Gabor's main idea was to retrieve the phase term $\Delta \phi(r)$, which in electron microscopy may take different forms, depending on the parameters to be studied from the specimen. Today, EH provides a unique phase- imaging approach for characterizing nano scale electrostatic and magnetic fields. Quantitative whole field measurements can be directly related to specific specimen features from the relative phase shifts between the electron wave that passed through the specimen and the reference electron beam. In particular, the phase recovered by $\mathrm{EH}$ is due to several contributions [82]:

$$
\Delta \phi(\boldsymbol{r})=\phi_{C}+\phi_{M}+\phi_{E}+\phi_{G}
$$

where each phase sub index stands for Crystalline, 


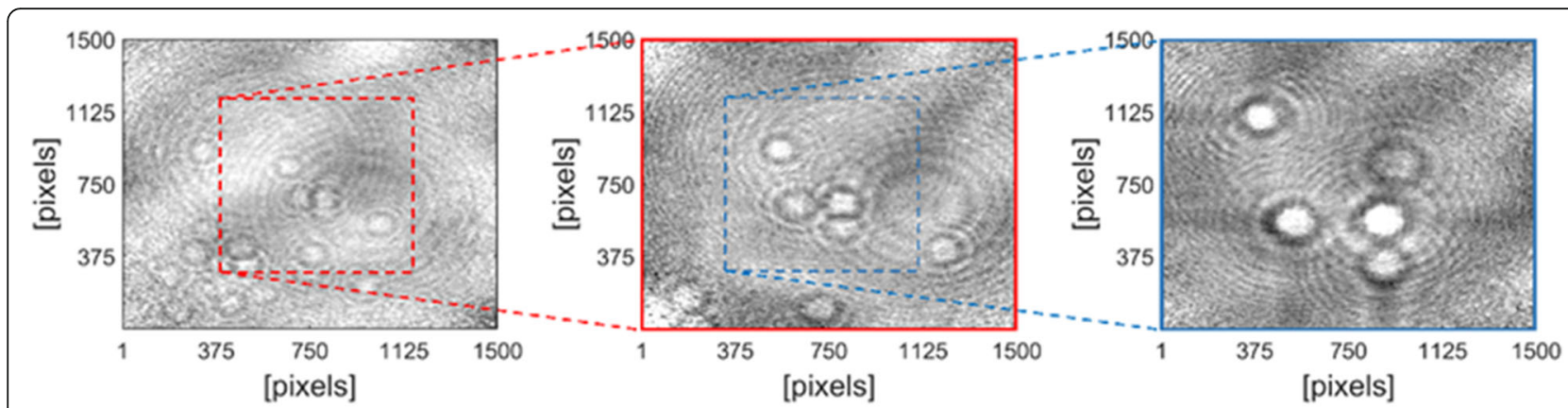

(a)

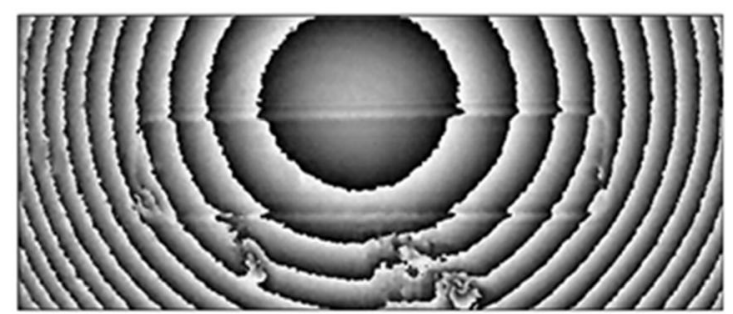

(b)

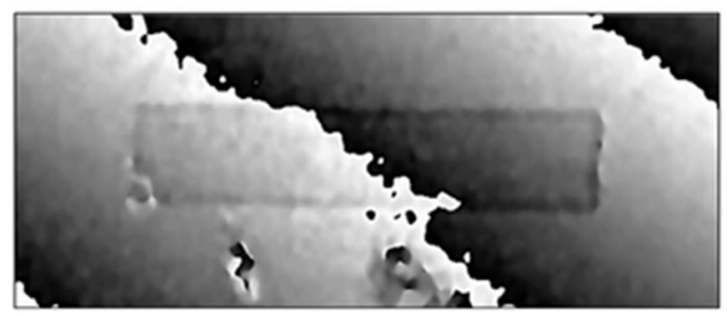

(c)

Fig. 24 a Example of three different FOV using the variable magnification and keeping the same camera's resolution, retrieved wrapped phase map for a standard calibration pattern, using either the (b) geometrical or the (c) controlled magnification. Reprinted from [67]. Copyright [2019], OSA

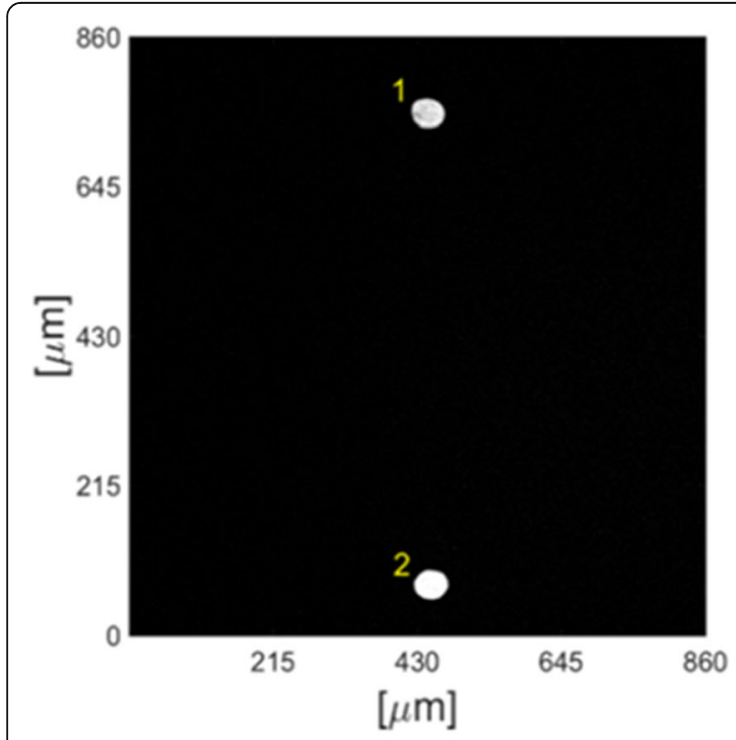

(a)

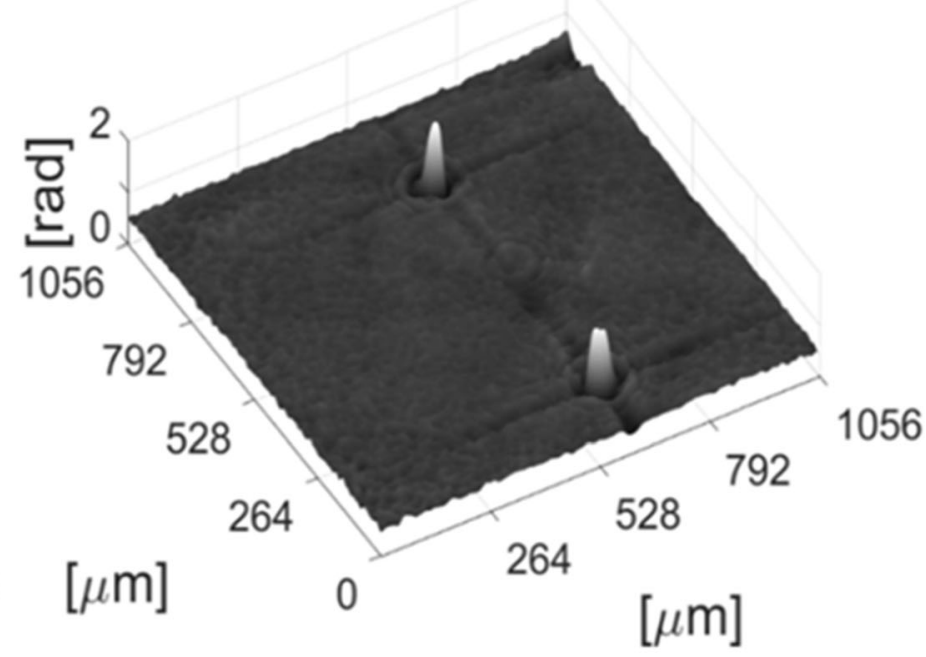

(b)

Fig. 25 (a) Confocal and (b) phase magnitude example of pollen particle visualization. Reprinted from [67]. Copyright [2019], OSA 

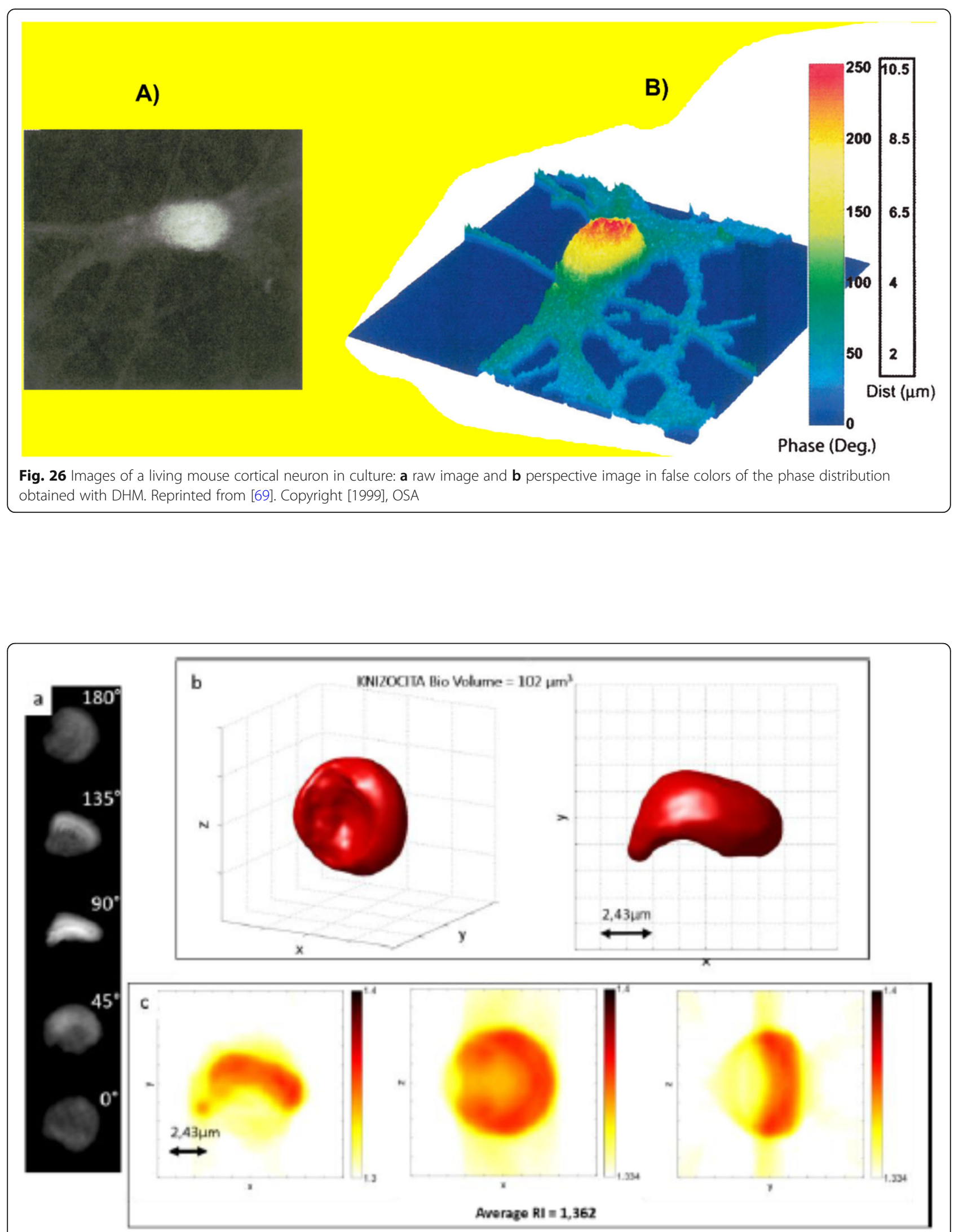

Fig. 27 Tomography images for rolling Red Blood Cell with a Knizocite shape. Reprinted from [71]. Copyright [2019], SPIE 


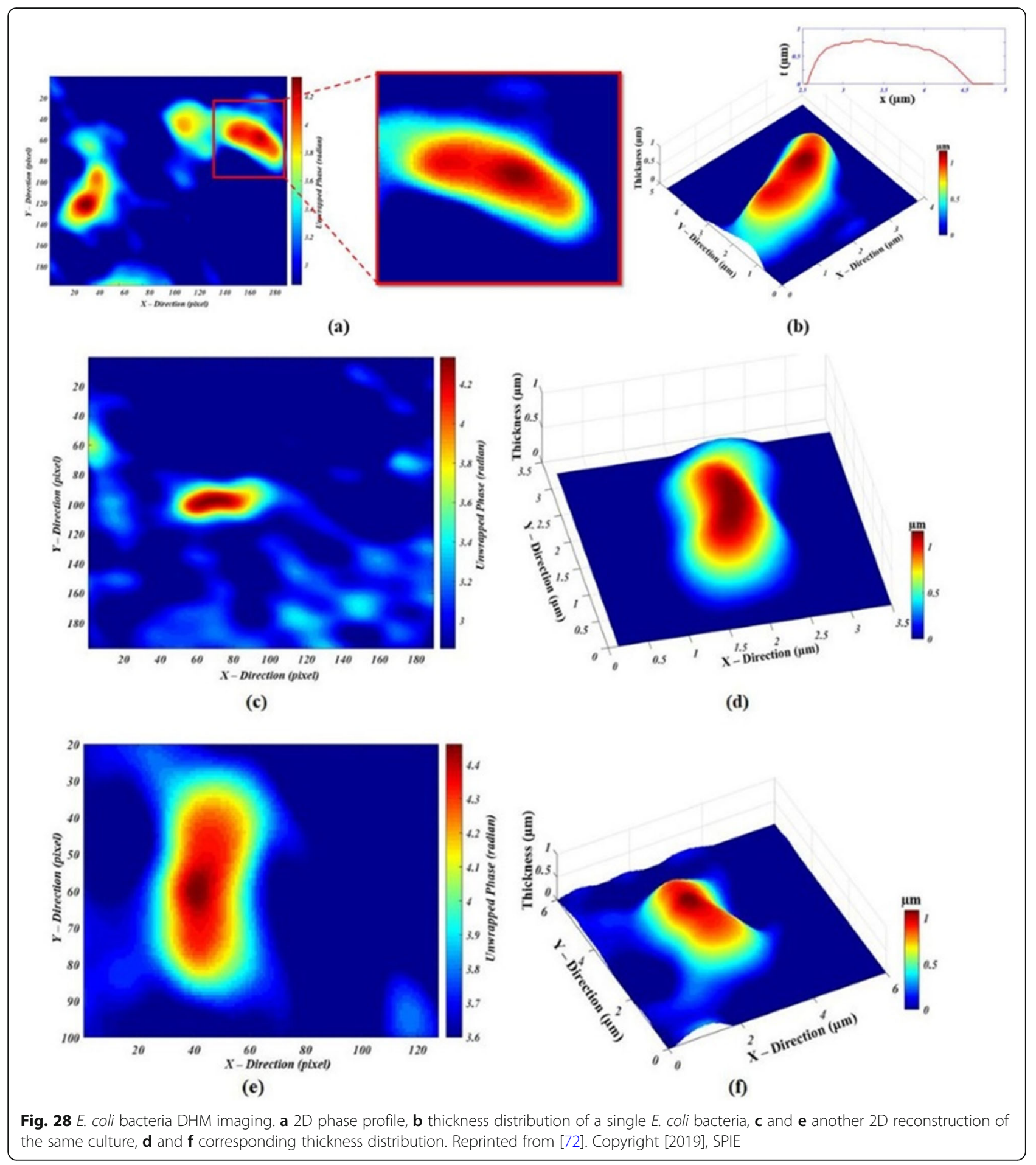

Magnetostatic, Electrostatic and Geometric respectively. The crystalline phase shift is expressed by [82, 83],

$$
C_{E}=\left(\frac{\pi}{\lambda \varepsilon}\right) \equiv \frac{2 \pi}{\lambda}\left(\frac{\varepsilon_{k}+\varepsilon_{0}}{\varepsilon_{k}\left(\varepsilon_{k}+2 \varepsilon_{0}\right)}\right)
$$

$$
\phi_{c}(x, y)=C_{E} \int V_{E}(x, y, z) \cdot d z
$$

with,

where $V_{E}$ is the electric potential, and $C_{E}$ is the electron interaction constant; $\varepsilon$ is the total energy of the electron, which depends on $\varepsilon_{0}$ the energy of the electron 

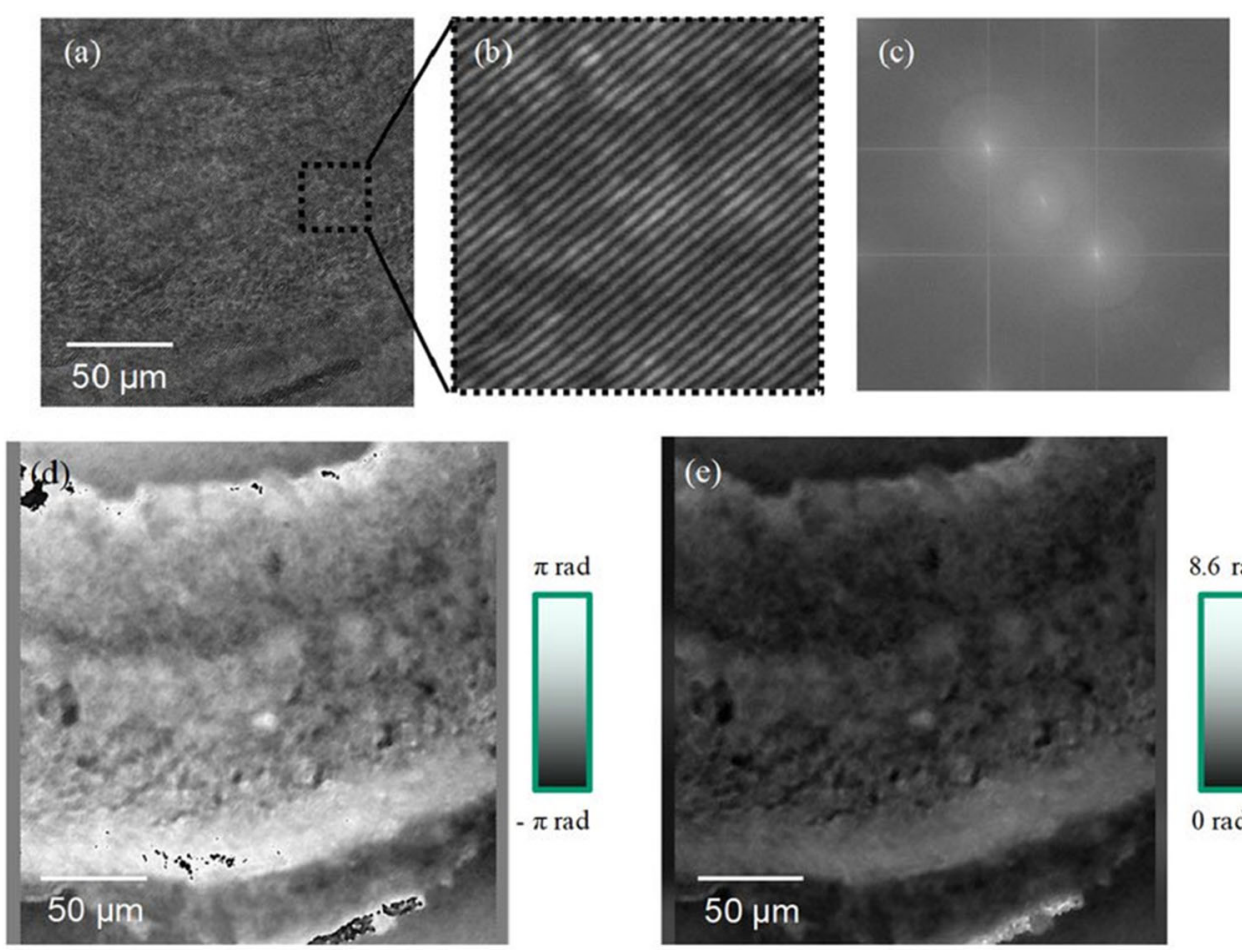

$8.6 \mathrm{rad}$

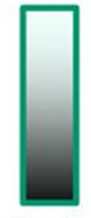

$0 \mathrm{rad}$

(f)

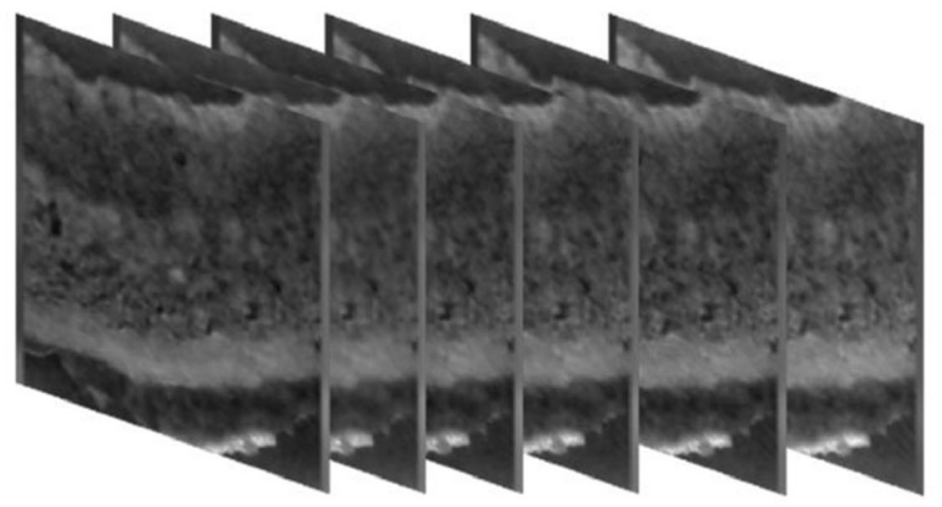

(g)

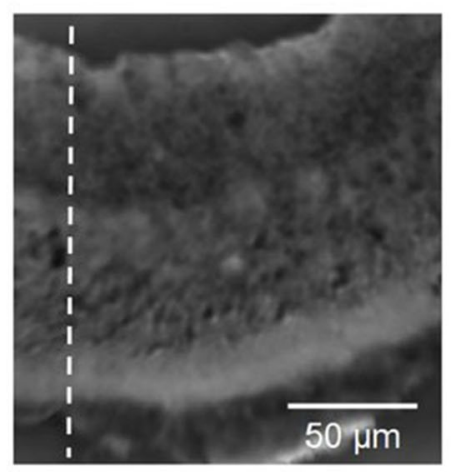

$\ldots \lambda=800 \mathrm{~nm}, \lambda=810 \mathrm{~nm}, \lambda=820 \mathrm{~nm}, \lambda=830 \mathrm{~nm}, \lambda=840 \mathrm{~nm}, \lambda=850 \mathrm{~nm}, \ldots$

Fig. 29 Multi-wavelength DHM images of dissected mouse retina: a digital off-axis holograms of a mouse retina section, $\mathbf{b}$ enlarged spatial carrier fringe pattern of $\mathbf{a}$; $\mathbf{c}$ its Fourier spectrum; $\mathbf{d}$ quantitative phase contrast image; e unwrapped phase; $\mathbf{f}$ stack of unwrapped phase images at different wavelengths in the same region of the retina; $\mathbf{g}$ averaged phase imaging of the stack of the unwrapped images, where the dashed line is a cross section for refractive index determination. Reprinted from [73]. Copyright [2019], SPIE

at rest and $\varepsilon_{k}$ the electron's kinetic energy, and electron wavelength.

The Geometric phase shift is [83],

$$
\phi_{G}(x, y)=-2 \pi g \cdot r
$$

where $g$ represents the reciprocal lattice vectors of the perfect, or "reference", crystal, and $\boldsymbol{r}$ is a point on the $(x, y)$ plane.

In general terms, the main component of the phase shift from a standard material (such as ferroelectric or magnetic) may be described as the sum of the electrostatic $\left(\phi_{E}\right)$ and the magnetostatic $\left(\phi_{M}\right)$ phases as follows [83]:

$$
\begin{aligned}
& \phi_{E}(x, y)=C_{E} \int E(x, y, z) \cdot d x \cdot d z \\
& \phi_{M}(x, y)=-\frac{e}{\hbar} \iint B_{\perp}(x, y) \cdot d x \cdot d z
\end{aligned}
$$

where $e$ is the electron charge, $E$ is the electric field 


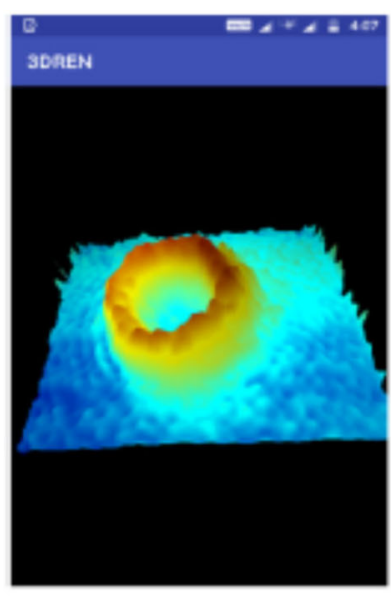

a)

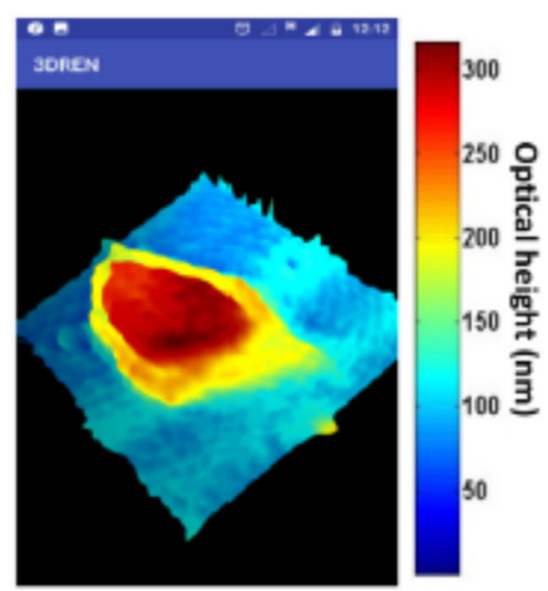

b)

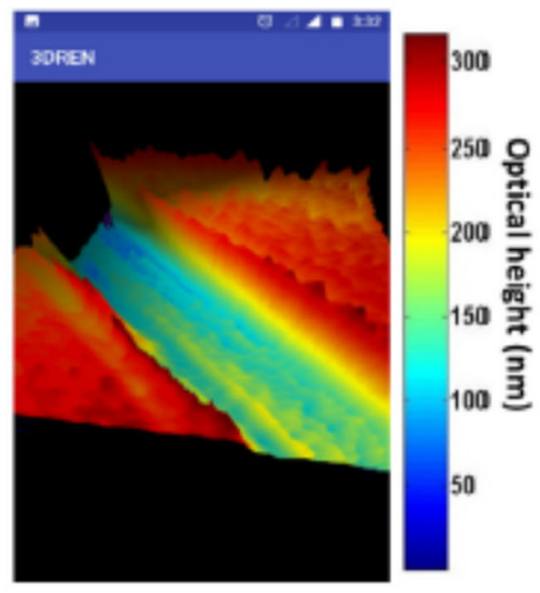

c)

Fig. 30 Screen shots of: a optical thickness variation of a Red Blood Cells (RBC) rendered on the smart phone; b optical thickness variation of HeLa cell rendered on the same device; and c surface profile of micro channel on aluminum coated silicon substrate rendered on smart phone. Reprinted [74]. Copyright [2019], Elsevier

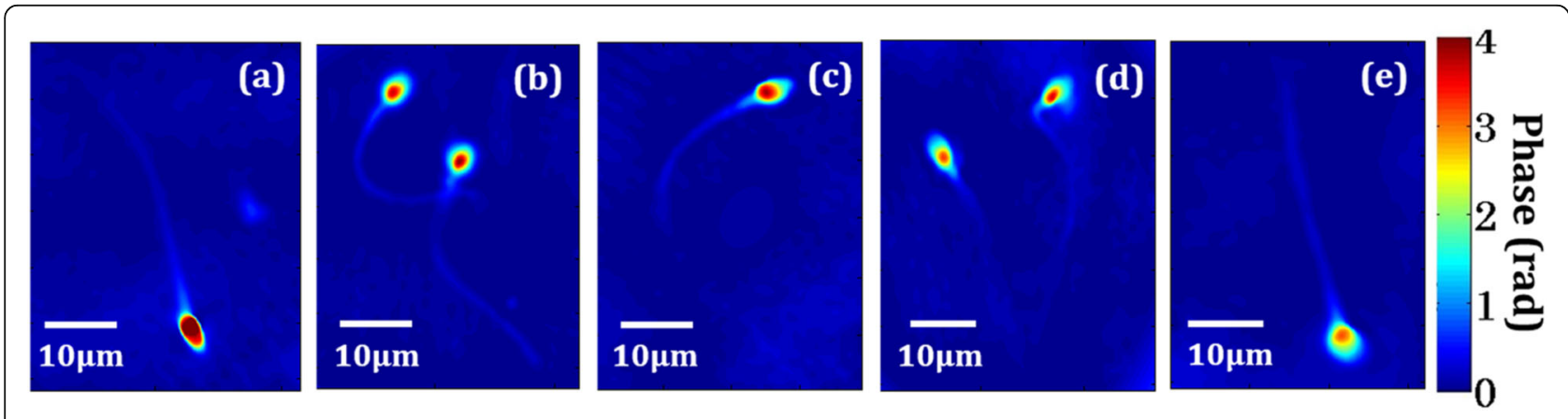

Fig. 31 DHM pseudo-color plot imaging of reconstructed phase maps of a) normal sperm cells and at different concentrations of $\mathrm{H}_{2} \mathrm{O}_{2}$ : $\mathbf{b}$ ) $10 \mu \mathrm{M}, \mathbf{c}) 40 \mu \mathrm{M}$, d) $70 \mu \mathrm{M}$ and e) $100 \mu \mathrm{M}$. Reprint from [75]. Copyright [2019], Science 


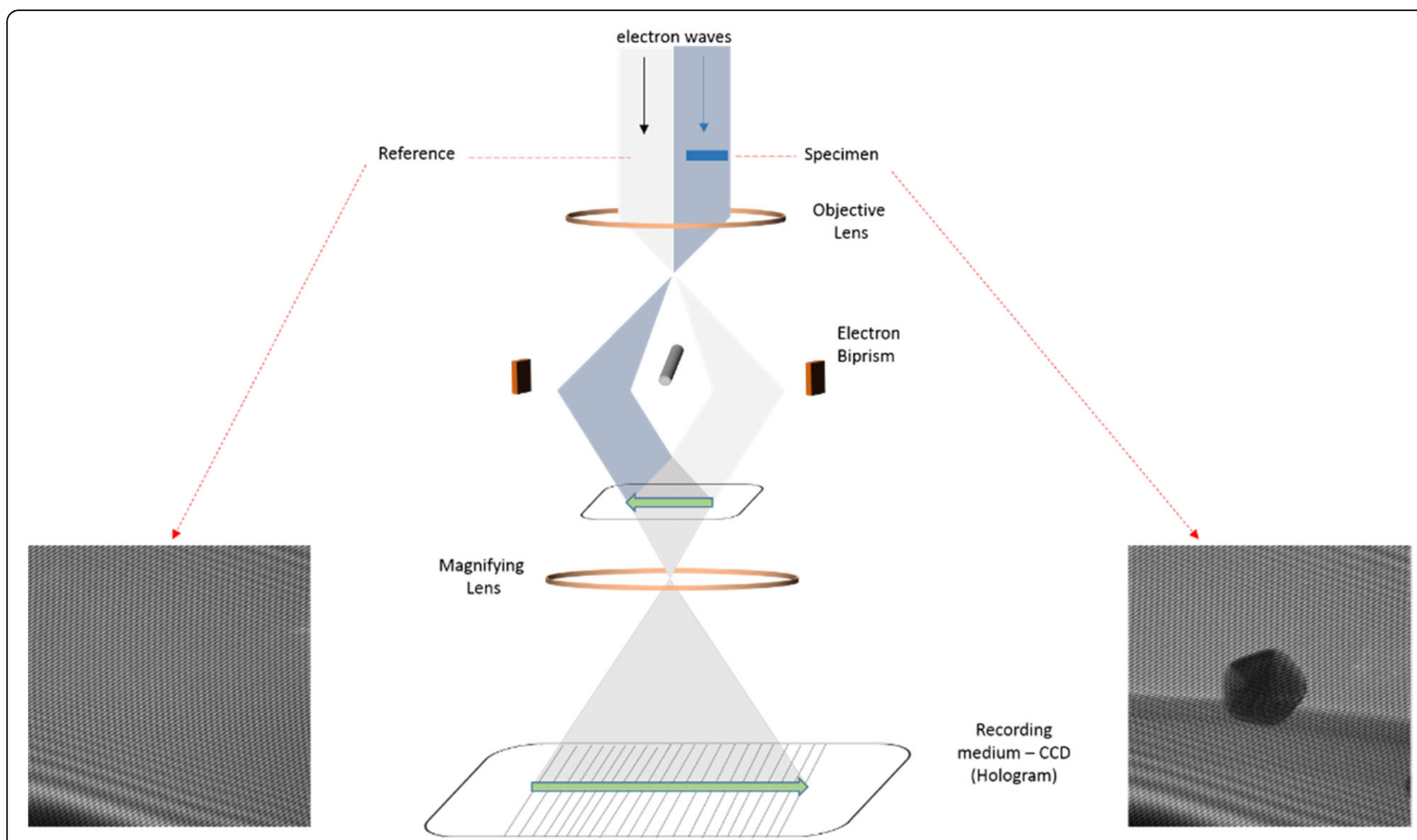

Fig. 32 Off-axis EHI schematic view. Reprint from [84]. Copyright [2014], ELSEVIER

associated to the electric potential $V_{E}(x, y, z)$, and $B$ is the magnetic field. Here $\phi_{E}$ represents the electrostatic component that may include the mean inner potential and induced polarization. The main aim for EHI is thus the recovery of the phase term $\Delta \phi(r)$, since the amplitude of the interferometric process may be read directly from counting the number of resulting fringes, whose separation is given by the electron's wavelength $\lambda$. In EHI the phase retrieval is done based on a Fourier procedure developed independently by several authors [9-11] and discussed previously.

\section{State of the art results in EHI}

An electron hologram is a fringe-modulated image containing the amplitude and the phase of an electron transparent object. These holograms are collected by the interference between the reference and the object wave, due to a biased electron biprism (schematically shown in Fig. 32). In this configuration, the sample is placed in a position that covers the wavefront interfering with the sample (object hologram) and the vacuum. In addition, a reference hologram (no sample) is recorded separately for a precise phase reconstruction [84]. After the acquisition, the holograms are numerically reconstructed into complex images where it is possible to extract both: the amplitude and the phase image.

The latter gives an extraordinary opportunity to study a wide variety of physical parameters in-on and around the specimen. Next, a couple of examples are given that involve the shape quantification of gold $(A u)$ nano particles [85] and the 3D visualization of a bacteria [86].

For a gold decahedral nano particle, Fig. 33 (a) and (b) show (after reconstruction) the wrapped and unwrapped phase images. The oriented particle shows a smooth contour moving from the center to the edge of the nano particle. In Fig. 33 (b) the unwrapped phase image is calibrated using the mean inner potential of the FCC (FFC: face-centered cubic) $A u$ nano particle. Considering the background signal as the reference plane, the thickness profile can be computed. Fig. 33d shows a thickness line profile from the line indicated in figure (b). The phase image shown in Fig. 33c has been amplified 2.5 times [85] from that shown in Fig. 33a, this just for illustration purposes.

Biological samples studied by conventional electron microscopy (scanning and transmission modes) need to be negatively stained or coated with metals to enhance their contrast, a procedure that may affect cellular components making that the interpretation- 

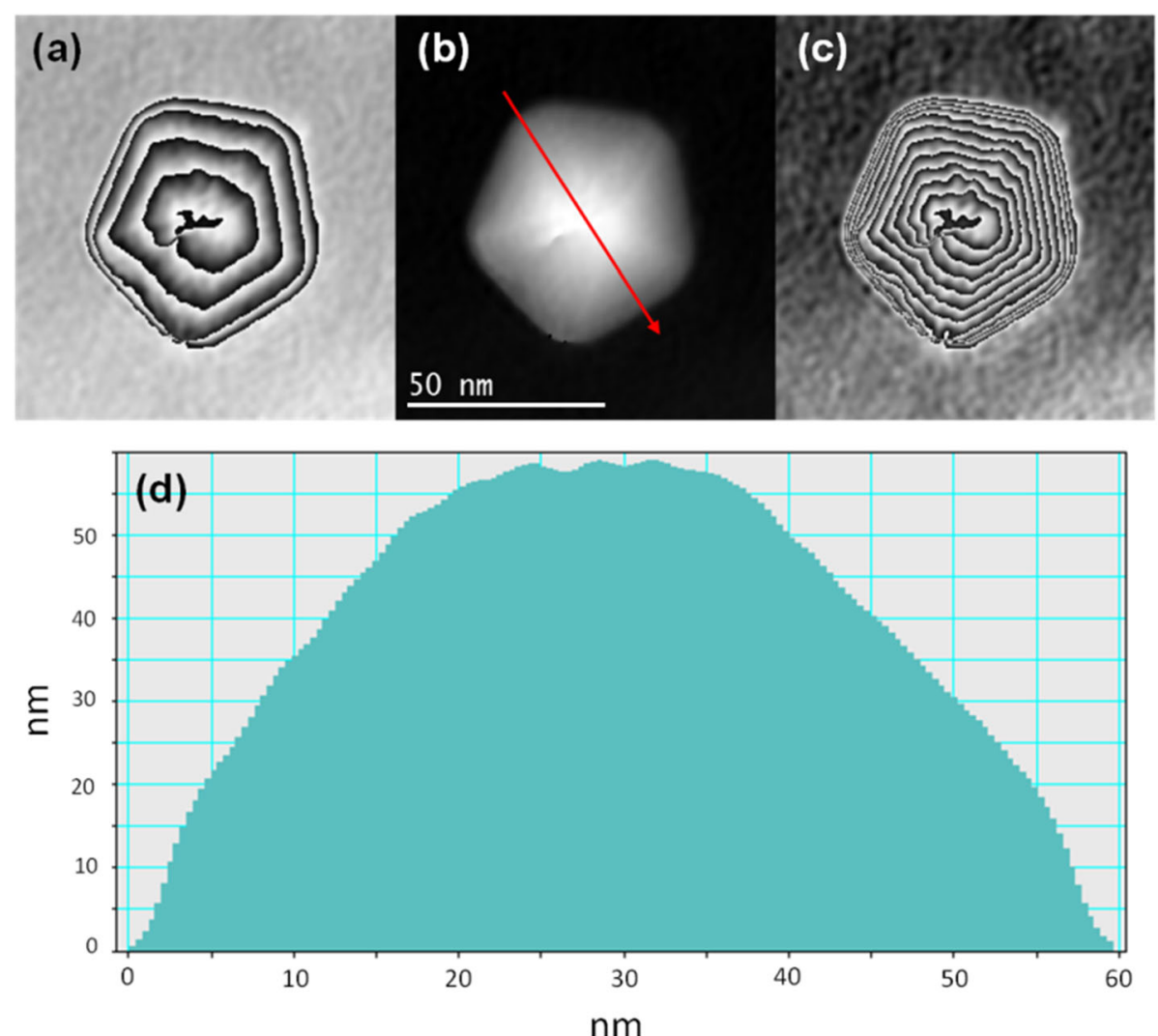

Fig. 33 Au decahedral nano particle: (a) wrapped phase image, (b) unwrapped phase image, (c) 2.5 times magnification of the original phase lines in figure (a), and (d) computed thickness obtained using the Au crystalline potential. Reprint from [85]. Copyright [2013], ELSEVIER

analysis process causes the formation of structural artifacts. Another limitation of electron microscopy in biological samples is the radiation damage, which in most cases also poses an additional challenge. In comparison, electron holography has a tremendous potential to recover sample information avoiding both problems since their structural information can be extracted even at low voltages, under cryo-conditions and at low doses. Particularly, electron holography is a highly sensitive imaging technique even for light atoms, able to discriminate chemical elements with similar atomic number and detect structures that contain different electrical potentials.

As an example, we present the Staphylococcus aureus (S. aureus) which is a gram $(+)$ bacteria of serious concern for public health. S. aureus infections, including methicillin-resistant $S$. aureus (MRSA) strains cause several diseases including healthcareassociated infections and wound infections that could derive in pneumonia, bloodstream infections and sepsis. S. aureus is usually found in the respiratory tract and skin. The bacteria was treated with avidin-streptavidin anti-S. aureus conjugated gold nanoparticles in order to confirm the specificity of
Ab-functionalized (antibody functionalized) $A u$ nano particles. To gain a better understanding of the location and effects of Ab-functionalized $A u$ nano particles on $S$. aureus off-axis electron holographic interferometry (EHI) was used to determine the 3D sample structure and the location of particles attached on cell walls. The three dimensional reconstruction can be achieved by acquiring several holograms from different observation or electron illumination positions [86]. A set of holograms were acquired by tilting the sample from $-30^{\circ}$ to $+30^{\circ}$ in steps of $15^{\circ}$ as shown in Fig. 34 (a). The resulting phase maps for each inclination are displayed in Fig. 34 (b) and their respective unwrapped phase maps are shown in Fig. 34(c). The series of unwrapped phase maps in (c) shows a phase variation from each other which is clearly due to the different orientation of the bacteria.

From the unwrapped phase maps the surface plot may be extracted and represented as the real 3D image of the complete bacteria surface contour. Different views from the surface plots are shown in Fig. 35, from which it is possible to distinguish the surface morphology of the bacteria cell wall showing 


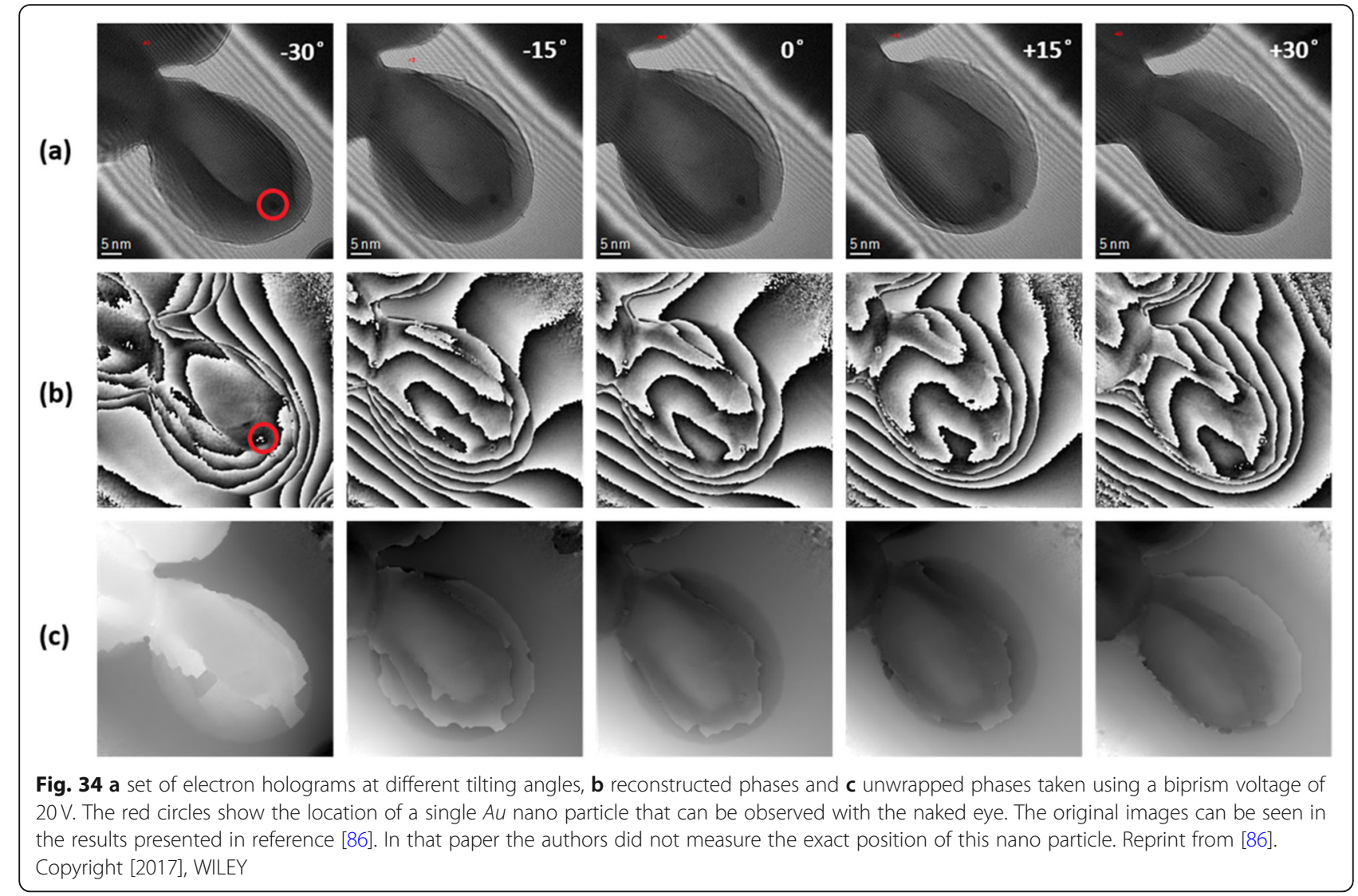

the prominent wave-like surface patterns. One advantage of $\mathrm{EHI}$ is the acquisition of surface information from a single specimen exposure and not by tilting the sample to reconstruct the surface of the samples.

\section{Conclusions}

DHI is a non-invasive optical metrology technique that has been successfully used for over 50 years in many different applications. With new advances in optomechatronic technologies and high-speed processors (in particular), DHI will definitely overcome and substitute the conventional metrology tools used. The technique is able to measure in 2D and 3D hidden object areas and can be taken outside laboratory controlled environments. It is ideally suited to study non-repeatable events from samples with sizes of a few meters to nanometers.

The technique was tested for different samples from nano to macro world, and the data obtained can be related to the type of tissue properties, like its density, rigidity, thickness, healthy or unhealthy condition, morphology, and refraction index. By means of the quantification of
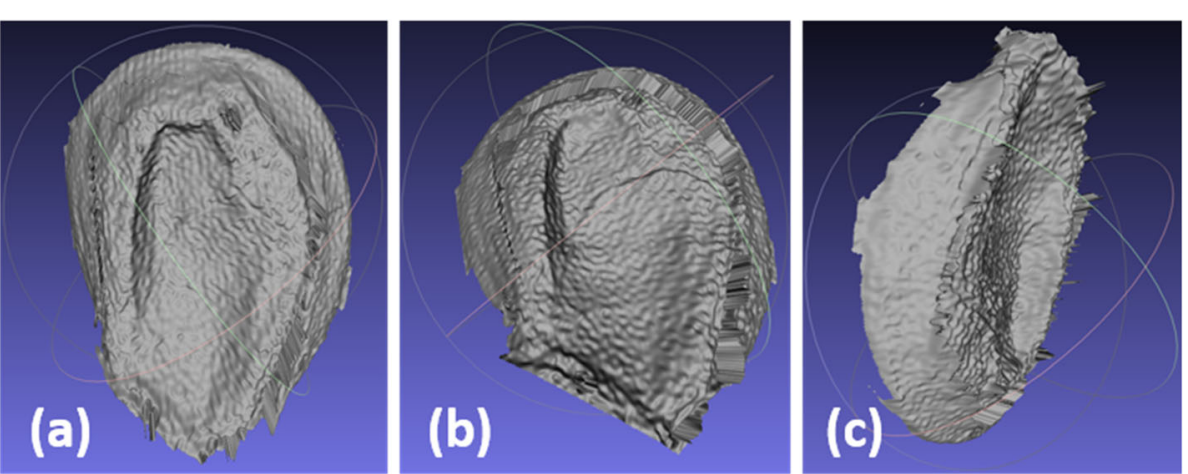

Fig. 35 Surface plot of the reconstructed phase showing a 3D view of the bacteria obtained by EHI from three different views: a zero tilting, $\mathbf{b}$ vertical tilting and c horizontal tilting. Reprint from [86]. Copyright [2017], WILEY 
displacement and imaging a characteristic unwrapped phase maps, one might associate it with the biomechanical condition or inside changes. DHI represents potentially an alternative and complementary tool capable of providing qualitative and quantitative new data which will be useful to improve the understanding of the behavior of the samples. The manuscript reports on a continuous increase in biological inspections using DHI with photons and electrons. This tendency is far to be reduced and more applications are reported, as the optical systems became more complex and sophisticated. Higher speeds and resolutions will allow future inspections as the on-board processing which uses new generation hardware for real-time inspections.

\section{Acknowledgements}

Not applicable.

\section{Authors' contributions}

All authors contributed equally in searching the data for this review. SHM and FMS, were the major contributors in writing the manuscript. The authors read and approved the final manuscript.

\section{Availability of data and materials}

Not Applicable.

\section{Competing interests}

The authors declare that they have no competing interests.

\section{Author details}

'Centro de Investigaciones en Óptica A. C, (CIO), Loma del Bosque 115, León, 37150 Guanajuato, Mexico. ${ }^{2}$ Centro de Investigaciones en Óptica A. C, (CIO), Unidad Aguascalientes, Prol. Constitución 607, Fracc. Reserva Loma Bonita, 20200 Aguascalientes, Ags, Mexico.

Received: 14 January 2020 Accepted: 23 April 2020

Published online: 29 June 2020

\section{References}

1. Powell, R.L., Stetson, K.A.: Interferometric vibration analysis by Wavefront reconstruction. J. Opt. Soc. Am. 55, 1593-1598 (1965)

2. Goodman, J.W., Lawrence, R.W.: Digital image formation from electronically detected holograms. Appl. Phys. Lett. 11, 77-79 (1967)

3. Lesem, L.B., Hirsch, P.M., Jordan J.., J.A.: Scientific applications: computer synthesis of holograms for 3-D display. Commun. ACM. 11, 661-674 (1968)

4. Brown, B.R., Lohmann, A.W.: Complex spatial filtering with binary masks. Appl. Optics. 5, 967-969 (1966)

5. Heflinger, L.O., Wuerker, R.F., Brooks, R.E.: Holographic interferometry. J. Appl. Phys. 37, 642-649 (1966)

6. Schnars, U.: Direct phase determination in hologram interferometry with use of digitally recorded holograms. JOSA A. 11, 2011-2015 (1994)

7. Schnars, U., Kreis, T.M., Jueptner, W.P.: Digital recording and numerical reconstruction of holograms: reduction of the spatial frequency spectrum. Optim. Eng. 35, 977-982 (1996)

8. Schnars, U., Juptner, W.: Direct recording of holograms by a CCD target and numerical reconstruction. Appl. Optics. 33(2), 179-181 (1994)

9. Takeda, M., Ina, H.I., Kobayashi, S.: Fourier-transform method of fringepattern analysis for computer-based topography and interferometry. JOSA. 72, 156-160 (1982)

10. Mendoza Santoyo, F., Kerr, D., Tyrer, J.R.: Manipulation of the Fourier components of speckle fringe patterns as part of an interferometric analysis process. J. Mod. Opt. 36, 195-204 (1989)

11. Yamaguchi, I.: Fringe formation in speckle photography. JOSA. A1, 81-86 (1984)

12. Boone, P.M.: NDT techniques: laser-based. In: Jürgen Buschow, K.H., Cahn Robert, W., Flemings Merton, C., Bernhard, I., Kramer Edward, J., Mahajan, S.,
Veyssière, P. (eds.) Encyclopedia of Materials: Science and Technology, pp. 6018-6021. Elsevier, Amsterdam (2001)

13. Vest, C:: M: Holographic Interferometry. Wiley, New York (1979)

14. Sharpe, W.N.: Springer Handbook of Experimental Solid Mechanics. SpringerVerlag, New York (2008)

15. Uribe, L.U., Hernández-Montes, M.S., Mendoza, S.F.: Fully automated digital holographic interferometer for 360 deg contour and displacement measurements. Optim. Eng. 55(12), 121719 (2016)

16. Hariharan, P., Oreb, B.: Stroboscopic holographic interferometry: application of digital techniques. Opt. Commun. 59(2), 83-86 (1986)

17. Pedrini, G., Gusev, M., Schedin, S., Tiziani, H.: Pulsed digital holographic interferometry by using a flexible fiber endoscope. Opt. Lasers Eng. 40(5-6), 487-499 (2003)

18. Pedrini, G., Schedin, S., Alexeenko, I., Tiziani, H.: Use of endoscopes in pulsed digital holographic interferometry. In: Hoefling, P., Jueptner, W.P.O., Kujawinska, M. (eds.) Proceeding of SPIE 4399, pp. 1-8 (2001)

19. Ferraro, P., De Nicola, S., Finizio, A., Grilli, S., Pierattini, G.: Digital holographic interferometry for characterization of transparent materials. In: Hoefling, R., Jueptner, W.P., Kujawinska, M. (eds.) Proceedings of SPIE 4399, pp. 9-16 (2001)

20. Owen, R., Zozulya, A.: Comparative study with double-exposure digital holographic interferometry and a shack-Hartmann sensor to characterize transparent materials. Appl. Optics. 41(28), 5891-5895 (2002)

21. De la Torre, I., Manuel, H., Mendoza Santoyo, F., Hernandez, M.M.S.: Transmission out-of-plane interferometer to study thermal distributions in liquids. Opt. Lett. 43, 871-874 (2018)

22. Agarwal, S., Kumar, M., Kumar, V., Shakher, C.: Analysis of alcohol-water diffusion process using digital holographic interferometry. In: Mendoza Santoyo, F.R., Mendez, E. (eds.) Proceedings of SPIE 9660 (2015)

23. Wang, J., Zhao, J., Di, J., Rauf, A., Hao, J.: Dynamically measuring unstable reaction-diffusion process by using digital holographic interferometry. Opt Lasers Eng. 57, 1-5 (2014)

24. Wylock, C., Dehaeck, S., Cartage, T., Colinet, P., Haut, B.: Experimental study of gas-liquid mass transfer coupled with chemical reactions by digital holographic interferometry. Chem. Eng. Sci. 66(14), 3400-3412 (2011)

25. De la Torre-lbarra, M.H., Mendoza Santoyo, F.: Interferometric study on birds' feathers. J. Biomed. Opt. 18(5), 1-9 (2013)

26. Aguayo, D., Mendoza Santoyo, F., De la Torre-I, M.H., Salas-Araiza, M.D., Caloca-Mendez, C., Gutierrez Hernandez, D.A.: Insect wing deformation measurements using high speed digital holographic interferometry. Opt. Express. 18, 5661-5667 (2010)

27. Aguayo, D., Santoyo, F.M., De la Torre Ibarra, M., Mendez, C.C., Salas-Araiza, M.D.: Comparison on different insects' wing displacements using high speed digital holographic interferometry. J. Biomed. Opt. 16, 1-9 (2011)

28. Silva, A.L., Hernández, M., del Socorro Mendoza Santoyo, M., De la Torre, I.M.H., Flores Moreno, J.M., Frausto, R., et al: Study of skin rigidity variations due to UV radiation using digital holographic interferometry. Opt. Lasers Eng. 126, 105909 (2020)

29. Zak, M., Kuropka, P., Kobielarz, M., Dudek, A., Kaleta-Kuratewicz, K., Szotek, S.: Determination of the mechanical properties of the skin of pig foetuses with respect to its structure. Acta Bioeng. Biomech. 13, 37-43 (2011)

30. Agache, P.G., Monneur, C., Leveque, J.L, De Rigal, J.: Mechanical properties and young's modulus of human skin in vivo. Arch. Dermatol. Res. 269, 221-232 (1980)

31. Li, C., Guan, G., Reif, R., Huang, Z., Wang, R.K.: Determining elastic properties of skin by measuring surface waves from an impulse mechanical stimulus using phase-sensitive optical coherence tomography. J. R. Soc. Interface. 9, 831-841 (2012)

32. Imokawa, G., Ishida, K.: Biological mechanisms underlying the ultraviolet radiation-induced formation of skin wrinkling and sagging l: reduced skin elasticity, highly associated with enhanced dermal elastase activity, triggers wrinkling and sagging. Int. J. Mol. Sci. 16, 7753-7775 (2015)

33. Takema, Y., Yorimoto, Y., Kawai, M., Imokawa, G.: Age-related changes in the elastic properties and thickness of human facial skin. Br. J. Dermatol. 131, 641-648 (1994)

34. Tilleman, T., Tilleman, M., Neumann, M.: The elastic properties of cancerous skin: Poisson's ratio and Young's modulus. IMAJ. 6, 753-755 (2004)

35. Tonndorf, J., Khanna, S.M.: Tympanic-membrane vibrations in human cadaver ears studied by time-averaged holography. J. Acoust. Soc. Am. 52, 1221-1233 (1972)

36. Decraemer, W.F., Khanna, S.M., Funnell, W.R.J.: Interferometric measurement of the amplitude and phase of tympanic membrane vibrations in cat. Hear. Res. 38, 1-17 (1989) 
37. Cheng, J.T., Aarnisalo, A.A., Harrington, E., del Hernandez-Montes, M.S., Furlong, C., Merchant, S.N., et al.: Motion of the surface of the human tympanic membrane measured with stroboscopic holography. Hear. Res. 263, 66-77 (2010)

38. Hernández-Montes, M. del S., Furlong, C., Rosowski, J.J., Hulli, N., et al.: Optoelectronic holographic otoscope for measurement of nanodisplacements in tympanic membranes. J. Biomed. Opt. 14(3), 034023 (2009)

39. Flores-Moreno, J.M., Furlong, C., Cheng, J.T., Rosowski, J.J., Merchant, S.N.: Characterization of acoustically induced deformations of human tympanic membranes by digital holography and shearography. In: Rodríguez-Vera, R., Díaz-Uribe, R. (eds.) Proceedings of SPIE 80118C-80118C - 10 (2011)

40. Cheng, J.T., Hamade, M., Merchant, S.N., Rosowski, J.J., Harrington, E., Furlong, C.: Wave motion on the surface of the human tympanic membrane: holographic measurement and modeling analysis. J. Acoust. Soc. Am. 133, 918-937 (2013)

41. Flores-Moreno, J.M., Mendoza Santoyo, F., Estrada Rico, J.C.: Holographic otoscope using dual-shot-acquisition for the study of eardrum biomechanical displacements. Appl. Optics. 52, 1731-1742 (2013)

42. Rosowski, J.J., Dobrev, I., Khaleghi, M., Lu, W., Cheng, J.T., Harrington, E., et al.: Measurements of three-dimensional shape and sound-induced motion of the chinchilla tympanic membrane. Hear. Res. 301, 44-52 (2013)

43. Khaleghi, M., Furlong, C., Ravicz, M., Cheng, J.T., Rosowski, J.J.: Threedimensional vibrometry of the human eardrum with stroboscopic lensless digital holography. J. Biomed. Opt. 20, 051028 (2015)

44. Razavi, P., Dobrev, I., Ravicz, M.E., Cheng, J.T., Furlong, C., Rosowski, J.J.: Transient response of the eardrum excited by localized mechanical forces. Mech. Biol. Syst. Mater. 6, 31-37 (2016)

45. Pedrini, G., Osten, W., Gusev, M.E.: High-speed digital holographic interferometry for vibration measurement. Appl. Optics. 45, 3456 (2006)

46. Solís, S.M., del Hernández-Montes, M.S., Santoyo, F.M.: Measurement of Young's modulus in an elastic material using 3D digital holographic interferometry. Appl. Optics. 50, 3383-3388 (2011)

47. Flores-Moreno, J.M., Furlong, C., Rosowski, J.J., Harrington, E., Cheng, J.T., Scarpino, C., et al.: Holographic otoscope for nanodisplacement measurements of surfaces under dynamic excitation. Scanning. 33, 342-352 (2011)

48. Hernandez-Montes, M., Mendoza Santoyo, F., Muñoz, S., Perez, C., De La Torre, M., Flores, M., Alvarez, L.: Surface strain-field determination of tympanic membrane using 3D-digital holographic interferometry. Opt. Lasers Eng. 71, 42-50 (2015)

49. Hernández-Montes, M., Mendoza Santoyo, F., Pérez López, C., Muñoz Solís, S., Esquivel, J.: Digital holographic interferometry applied to the study of tympanic membrane displacements. Opt. Lasers Eng. 49, 698-702 (2011)

50. Trillo, C., Doval, A.F., Hernández-Montes, S., Deán-Ben, X.L., López-Vázquez, J. C., Fernández, J.L.: Pulsed TV holography measurement and digital reconstruction of compression acoustic wave fields: application to nondestructive testing of thick metallic samples. Meas. Sci. Technol. 22, 025109 (2011)

51. M. Solís, S., del Hernández-Montes, M.S., M. Santoyo, F.: Tympanic membrane contour measurement with two source positions in digital holographic interferometry. Biomed. Opt. Express. 3, 3203-3210 (2012)

52. Solís, S., Santoyo, F., del Hernández-Montes, M.S.: 3D displacement measurements of the tympanic membrane with digital holographic interferometry. Opt. Express. 20(5), 5613-5621 (2012)

53. Santiago-Lona, C., del Hernández-Montes, M.S., F. Moreno, M., Piazza, V., De La Torre, M., Pérez-López, C., Mendoza-Santoyo, F., Sierra, A., Esquivel, J.: Tympanic membrane displacement and thickness data correlation using digital holographic interferometry and confocal laser scanning microscopy Optim. Eng. 58, 084106 (2019)

54. Kumar, A.: Small animal ear diseases. In: Gotthelf, L.N. (ed.) Anatomy of the Canine and Feline Ear. Elsevier Saunders, St. Louis (2005)

55. Hernández-Montes, M. del S., Muñoz, S., De La Torre, M., Flores, M., Pérez, C., Mendoza-Santoyo, F.: Quantification of the vocal folds' dynamic displacements. J. Phys. D: Appl. Phys. 49, 175401 (1-7) (2016)

56. Hernández-Montes, M. del S., Muñoz, S., Mendoza, F.: Measurement of vocal folds displacements using high-speed digital holographic interferometry. LAOP Technical Digest LTu4A. 28, (2014)

57. Pedrini, G., Alexeenko, I., Zaslansky, P., Tiziani, H.J., Osten, W.: Digital holographic interferometry for investigations in biomechanics. Proc. SPIE. 5776, 325-332 (2005)
58. Dirksen, D., Droste, H., Kemper, B., Deleré, H., Deiwick, M., Scheld, H., Von Bally, G.: Lensless Fourier holography for digital holographic interferometry on biological samples. Opt. Lasers Eng. 36, 241-249 (2001)

59. Akhmetshin, A.M.: High sensitive multiresolution analysis of low-contrast radiologic images based on the digital pseudo coherent holographic interferometry method. J. Digit. Imaging. 12(2 SUPPL. 1), 197-198 (1999)

60. Belashov, A.V., Belyaeva, T.N., Kornilova, E.S., Petrov, N.V., Salova, A.V., Semenova, I.V., Vasyutinskii, O.S., Zhikhoreva, A.A.: Detection of photoinduced transformations in live HeLa cells by means of digital holographic micro-interferometry. In: Imaging and Appl. Opt. DTh11.5 (2016)

61. Kumar, M., Singh Birhman, A., Kannan, S., Shakher, C.: Measurement of initial displacement of canine and molar in human maxilla under different canine retraction methods using digital holographic interferometry. Optim. Eng. 57 094106 (2018)

62. Tavera, R.C.G., De La Torre, I.M.H., Flores, M.J.M., Luna, H.J.M., Briones, R.M.J., Mendoza, S.F.: Optical phase analysis in drilled cortical porcine bones using digital holographic interferometry. In: Popescu, G., Park, Y. (eds.) Proceedings of SPIE, p. 9718 (2016)

63. Alvarez, A., De La Torre Ibarra, M., Santoyo, F., Anaya, T.-S.: Strain determination in bone sections with simultaneous 3D digital holographic interferometry. Opt. Lasers Eng. 57, 101-108 (2014)

64. Pantelić, D., Grujić, D., Vasiljević, D.: Single-beam, dual-view digital holographic interferometry for biomechanical strain measurements of biological objects. J. Biomed. Opt. 19, 127005 (2014)

65. Tavera, R.C.G., De la Torre, I.M.M.H.H., Flores, M.J.M., Hernandez, M., Ma Del, S. , Mendoza-Santoyo, F., Briones, R.M.J., Sanchez, P.J.: Surface structural damage study in cortical bone due to medical drilling. Appl. Optics. 56, F179-F188 (2017)

66. Tavera Ruiz, C.G., De La Torre-lbarra, M.H., Flores-Moreno, J.M., Frausto-Reyes, C., Mendoza Santoyo, F.: Cortical bone quality affectations and their strength impact analysis using holographic interferometry. Biomed. Opt. Express. 9, 4818-4833 (2018)

67. Frausto-Rea, G., De la Torre, M.H., Flores, J.M., Silva, L., Briones-R, M., Mendoza Santoyo, F.: Micrometric size measurement of biological samples using a simple and non-invasive transmission interferometric set up. Opt. Express. 27, 26251-26263 (2019)

68. Popescu, G.: Quantitative Phase Imaging of Cells and Tissues. McGraw-Hill Biophotonics. McGraw-Hill, New York (2011)

69. Marquet, P., Rappaz, B., Magistretti, P.J., Cuche, E., Emery, Y., Colomb, T., Depeursinge, C.: Digital holographic microscopy: a noninvasive contrast imaging technique allowing quantitative visualization of living cells with subwavelength axial accuracy. Opt. Lett. 30, 468-470 (2005)

70. Kim, M.K.: Digital Holographic Microscopy, Springer Series in Optical Sciences. Springer Science+Business Media, New York (2011)

71. Bianco, V., Miccio, L., Memmolo, P., Merola, F., Mandracchia, B., Cacace, T., et al.: 3D imaging in microfluidics: new holographic methods and devices. Microfluidics BioMEMS Med. Microsyst. XVII. Proc SPIE. 10875, 108750U (2019)

72. Rastogi, V., Gadkari, R., Agarwal, S., Dubey, S.K., Shakher, C.: Digital holographic interferometric in vitro imaging of Escherichia coli (E. coli) bacteria. Holography. 11030, 1103011 (2019)

73. Barroso Peña, Á., Ketelhut, S., Heiduschka, P., Nettels-Hackert, G., Schnekenburger, J., Kemper, B.: Refractive index properties of the retina accessed by multi-wavelength digital holographic microscopy. Proc. SPIE. 10883, 108830X (2019)

74. Goud, B.K., Shinde, D.D., Udupa, D.V., Krishna, C.M., Rao, K.D., Sahoo, N.K. Low cost digital holographic microscope for 3-D cell imaging by integrating smartphone and DVD optical head. Opt. Lasers Eng. 114, 1-6 (2019)

75. Dubey, V., Popova, D., Ahmad, A., Acharya, G., Basnet, P., Mehta, D.S., et al.: Partially spatially coherent digital holographic microscopy and machine learning for quantitative analysis of human spermatozoa under oxidative stress condition. Sci. Rep. 9, 3564 (2019)

76. Gabor, D.: Microscopy by reconstructed wavefronts. Proc. Royal Soc. London Ser. A. 197, 454 (1949)

77. Gabor, D.: Microscopy by reconstructed wavefronts: II. Proc. Soc. Phys. Soc B. 64,449 (1951)

78. Möllenstedt, G.: And Düker., H.: Beobachtungen und messungen an briprisma-interferenzen mit elektronenwellen. Z. Physik. 145, 377-397 (1956)

79. Tonomura, A., Endo, J., Matsuda, T.: An application of electron holography to interference microscopy. Optik. 53, 143 (1979)

80. Tonomura, A.: Appication of electron holography using a field-emission electron microscope. J. Electron Microsc. (Tokyo). 33, 101 (1984) 
81. Yatagai, T., Ohmura, K., Iwasaki, S., Hasegawa, S., Endo, J., Tonomura, A.: Quantitative phase analysis in electron holographic interferometry. Appl. Optics. 26, 377-382 (1987)

82. Völkl, E., Allard, L.F., Joy, D.C.: Introduction to Electron Holography. Springer Science + Business Media, New York (1999)

83. Hÿtch, M.J., et al.: Dark-field electron holography for the measurement of geometric phase. Ultramicroscopy. 111, 1328-1337 (2011)

84. Cantu-Valle, J., Ruiz-Zepeda, F., Mendoza-Santoyo, F., Jose-Yacaman, M., Ponce, A.: Calibration for medium resolution off-axis electron holography using a flexible dual-lens imaging system in a JEOL ARM 200F microscope. Ultramicroscopy. 147, 44-50 (2014)

85. Cantu-Valle, J., Ruiz-Zepeda, F., Voelkl, E., Kawasaki, M., Jose-Yacaman, M., Ponce, A.: Determination of the surface morphology of gold-decahedra nanoparticles using an off-axis electron holography dual-lens imaging system. Micron. 54-55, 82-86 (2013)

86. Ortega, E., Cantu-Valle, J., Plascencia-Villa, G., Vergara, S., Mendoza-Santoyo, F., Londono-Calderon, A., Santiago, U., Ponce Pedraza, A.: Morphology visualization of irregular shape bacteria by electron holography and tomography. Microsc. Res. Tech. 80(12), 1249-1255 (2017)

\section{Publisher's Note}

Springer Nature remains neutral with regard to jurisdictional claims in published maps and institutional affiliations.

\section{Submit your manuscript to a SpringerOpen ${ }^{\circ}$ journal and benefit from:}

- Convenient online submission

- Rigorous peer review

- Open access: articles freely available online

High visibility within the field

- Retaining the copyright to your article

Submit your next manuscript at $\boldsymbol{\nabla}$ springeropen.com 